\title{
Trivium
}

Revue franco-allemande de sciences humaines et sociales - Deutsch-französische Zeitschrift für Geistesund Sozialwissenschaften

32 | 2021

Institutions

\section{Die Soziologie: Gegenstand und Methode (1901)}

\section{Paul Fauconnet et Marcel Mauss}

Traducteur : Bernd Schwibs

\section{Q OpenEdition}

\section{Journals}

Édition électronique

URL : http://journals.openedition.org/trivium/7501

DOI : 10.4000/trivium.7501

ISBN : 1963-1820

ISSN : 1963-1820

\section{Éditeur}

Les éditions de la Maison des sciences de l'Homme

\section{Référence électronique}

Paul Fauconnet und Marcel Mauss, „Die Soziologie: Gegenstand und Methode (1901)“, Trivium [Online], 32 | 2021, online erschienen am 28 Januar 2021, abgerufen am 18 März 2021. URL: http:// journals.openedition.org/trivium/7501 ; DOI: https://doi.org/10.4000/trivium.7501

Ce document a été généré automatiquement le 18 mars 2021.

\section{(c) (i) (9)}

Les contenus des la revue Trivium sont mis à disposition selon les termes de la Licence Creative Commons Attribution - Pas d'Utilisation Commerciale - Pas de Modification 4.0 International. 


\title{
Die Soziologie: Gegenstand und Methode (1901)
}

\author{
Paul Fauconnet et Marcel Mauss
}

Traduction : Bernd Schwibs

\section{NOTE DE L'ÉDITEUR}

\section{Originalausgabe | édition originale}

"La Sociologie, objet et méthode«, La Grande Encyclopédie. Inventaire raisonné des sciences des lettres et des arts, Paris: Société anonyme de la Grande Encyclopédie, 1901, Bd. 30,

S. 165-176.

Die Originalausgabe ist in der Digitalen Bibliothek der Bibliothèque nationale de France (Gallica) zugänglich: https://gallica.bnf.fr/ark:/12148/bpt6k24665k.

1 Soziologie, ein von Auguste Comte geprägtes Wort zur Bezeichnung der Wissenschaft von den Gesellschaften. Obwohl aus einem lateinischen Stamm und einer griechischen Endung gebildet und deshalb lange Zeit von den Puristen abgelehnt, hat das Wort sich heute in allen europäischen Sprachen eingebürgert. Wir werden versuchen, nacheinander den Gegenstand der Soziologie und dann die von ihr verwendete Methode zu bestimmen. Danach verweisen wir auf die hauptsächlichen Gliederungen der unter diesem Namen sich konstituierenden Wissenschaft.

2 Man wird mühelos erkennen, dass wir uns unmittelbar von Ideen inspirieren lassen, die Durkheim in seinen verschiedenen Werken zum Ausdruck gebracht hat. Wir übernehmen sie übrigens nicht nur, weil sie uns aus theoretischen Gründen gerechtfertigt erscheinen, sondern weil sie unserem Eindruck nach die Grundsätze formulieren, deren sich die verschiedenen Sozialwissenschaften im Verlauf ihrer Entwicklung tendenziell immer bewusster werden. 


\section{Der Gegenstand der Soziologie}

3 Da neueren Ursprungs und kaum der philosophischen Periode entsprungen, wird der Soziologie immer noch die Existenz abgesprochen. Alle metaphysischen Traditionen, die den Menschen zu einem einzigartigen Wesen außerhalb der Natur stilisieren und in dessen Handlungen Tatsachen sehen, die sich grundlegend von den Naturtatsachen unterscheiden, sperren sich gegen die Fortschritte des soziologischen Denkens. Der Soziologe aber hat seine Forschungen nicht durch eine philosophische Argumentation zu begründen. Die Wissenschaft muss zu Werke gehen, sobald sie die Möglichkeit dazu bemerkt, und selbst traditionelle philosophische Theorien können keine Einwände gegen die Legitimität ihrer Vorgehensweisen darstellen. Wenn im Übrigen, was wahrscheinlich ist, sich aus der wissenschaftlichen Untersuchung der Gesellschaften zwingend eine andere Auffassung der menschlichen Natur ergibt, ist es an der Philosophie, sich mit der Wissenschaft und deren möglichen Ergebnissen abzustimmen. Die Wissenschaft aber hat diese fernen Folgen ihrer Entdeckungen weder vorherzusagen noch zu vermeiden.

Die Soziologie postuliert einzig und allein, dass die sogenannten sozialen Tatsachen innerhalb der Natur sind, das heißt, dass sie dem Prinzip der Ordnung und des Determinismus unterworfen, also beide gleichermaßen universell und infolgedessen verstehbar sind. Diese Hypothese ist nicht Resultat metaphysischer Spekulation, sondern ergibt sich aus einer Verallgemeinerung, die völlig legitim erscheint. Nach und nach ist diese aller Wissenschaft zugrunde liegende Hypothese auf alle Reiche erweitert worden, selbst auf jene, die sich am stärksten ihrem Zugriff zu entziehen schienen: Es ist von daher vernünftig, davon auszugehen, dass das Reich des Gesellschaftlichen - so es als Reich bezeichnet zu werden verdient - keine Ausnahme darstellt. Es obliegt nicht dem Soziologen zu beweisen, dass die sozialen Phänomene Gesetzen unterworfen sind: Es ist vielmehr an den Gegnern der Soziologie, den Gegenbeweis zu liefern. Denn a priori muss eingeräumt werden, dass das, was im Hinblick auf physische, biologische und psychische Phänomene als wahr entdeckt wurde, auch für die sozialen Tatsachen gilt. Nur ein definitives Scheitern könnte diese logische Vorannahme zunichtemachen. Doch von jetzt an ist dieses Scheitern nicht mehr zu befürchten. Die Behauptung, die Wissenschaft als ganze sei neu zu erfinden, ist nicht mehr haltbar. Wir wollen durchaus nicht die Bedeutung ihrer Ergebnisse überschätzen; doch ungeachtet aller skeptischen Einwände gibt es die Wissenschaft, und sie schreitet voran: Sie wirft klar definierte Probleme auf und weist zumindest auf Lösungen hin. Je stärker sie mit den Fakten in Kontakt gerät, umso mehr sieht sie, wie sich unvermutete Regelmäßigkeiten auftun, genauere Übereinstimmungen als zunächst erwartet; umso mehr verstärkt sich folglich das Gefühl, sich einer natürlichen Ordnung gegenüber zu finden, deren Existenz nur noch durch realitätsferne Philosophen in Frage gestellt werden kann.

5 Aber auch wenn ohne vorgängige Prüfung davon ausgegangen werden muss, dass die sogenannten sozialen Tatsachen natürlich, verstehbar und folglich wissenschaftliche Gegenstände sind, muss doch auch geklärt sein, dass es überhaupt Tatbestände gibt, die richtigerweise mit diesem Namen bezeichnet werden können. Damit sich eine neue Wissenschaft konstituiert, ist hinreichend, aber auch notwendig: zum einen, dass sie auf eine Ordnung von Tatsachen Anwendung findet, die klar von denjenigen unterschieden sind, mit denen sich die anderen Wissenschaften beschäftigen; zum anderen, dass diese Tatsachen sich unmittelbar verbinden lassen und sich wechselseitig 
erklären, ohne dass dafür andersgeartete Tatsachen herangezogen werden müssen. Denn eine Wissenschaft, die die Tatsachen, die ihren Gegenstand ausmachen, nur im Rückgriff auf eine andere Wissenschaft erklären könnte, würde sich mit Letzterer vermischen. Genügt die Soziologie dieser doppelten Voraussetzung?

\section{Vom sozialen Phänomen}

6 Gibt es spezifisch soziale Tatsachen? Das wird gemeinhin noch geleugnet, und unter den Leugnern sind sogar Denker, die ihrem Anspruch nach soziologisch arbeiten. Beispielhaft ist hier Tarde. Für ihn sind die sogenannten sozialen Tatsachen nichts als Ideen oder individuelle Gefühle, die sich durch Nachahmung verbreitet hätten. Ihnen käme mithin keinerlei spezifischer Charakter zu; denn eine Tatsache wechselt ihre Natur nicht schon dadurch, dass sie mehr oder weniger oft wiederholt wird. Für den Augenblick müssen wir diese Theorie nicht diskutieren; aber wir sollten festhalten, dass die Soziologie, wäre jene begründet, sich nicht von der Individualpsychologie unterscheiden würde, was hieße, dass jeglicher Stoff für eine genuine Soziologie fehlte. Sobald man die Besonderheit der sozialen Tatsachen verneint, zwingt sich der gleiche Schluss auf, ungeachtet der jeweiligen Theorie. Daran lässt sich die Bedeutung der von uns untersuchten Frage ermessen.

7 Ein erster Tatbestand bleibt sich immer gleich, nämlich dass es Gesellschaften gibt, das heißt Aggregate von menschlichen Wesen. Unter diesen Aggregaten sind einige von Dauer, wie die Nationen, andere vergänglich, wie Mengen bzw. Massen, die einen sind umfänglich, wie die großen Kirchen, die anderen sehr klein, wie die Familie, wenn sie auf ein Ehepaar beschränkt ist. Doch welche Größe und Form diese Gruppen und auflistbaren Phänomene wie Klasse, Stamm, Berufsgruppe, Kaste, Gemeinde haben mögen, alle zeichnen sich dadurch aus, dass sie aus einer Vielzahl individueller Bewusstseine gebildet werden, die aufeinander wirken und reagieren. Am Vorhandensein derartiger Aktionen und Reaktionen, dieser Interaktionen, erkennt man Gesellschaften. Die Frage ist nun, ob unter diesen Geschehnissen, die sich innerhalb der Gruppen vollziehen, welche sind, die die Beschaffenheit der Gruppe als Gruppe manifestieren und nicht nur die Beschaffenheit der sie ausmachenden Individuen, der allgemeinen Attribute der Menschheit. Sind darunter solche, die so sind, weil die Gruppe so ist, wie sie ist? Unter dieser - und nur dieser - Voraussetzung gibt es eine Soziologie im eigentlichen Sinn; denn dann gibt es ein Leben der Gesellschaft, das sich von dem unterscheidet, das die Individuen führen, oder vielmehr von dem, das sie führten, wenn sie isoliert lebten.

8 Nun gibt es durchaus realiter Phänomene mit derartigen Merkmalen, nur muss man sie entdecken können. Denn nicht alles, was sich in einer sozialen Gruppe ereignet, ist Erscheinungsform des Lebens der Gruppe als solcher und folglich sozial, so wie auch nicht alles, was sich in einem Organismus ereignet, genuin biologisch ist. Nicht nur die durch kosmische Ursachen bewirkten zufälligen und lokalen Störungen, sondern auch normale, regelmäßig wiederholte Ereignisse, die ausnahmslos alle Mitglieder einer Gruppe interessieren, können als nichtsoziale Tatsachen ausgezeichnet sein. So erfüllen alle Individuen, mit Ausnahme der Kranken, unter wahrnehmbar gleichen Bedingungen ihre organischen Funktionen. Dies gilt auch für die psychologischen Funktionen: Phänomene wie Empfindung, Vorstellen, Reaktion oder Hemmung sind bei allen Gruppenmitgliedern die gleichen. Sie sind bei allen denselben Gesetzen 
unterworfen, die die Psychologie erforscht. Doch trotz ihrer Allgemeinheit wird sie niemand als soziale Tatsachen kategorisieren. Der Grund dafür ist, dass sie nicht mit der Beschaffenheit der Gruppierung zusammenhängen, sondern sich aus der organischen und psychischen Natur des Individuums herleiten. Daher rührt auch ihre Gleichheit, ungeachtet der Gruppe, zu der das Individuum gehört. Wäre ein isolierter Mensch denkbar, könnte man sagen, dass sie selbst außerhalb der Gesellschaft sind, was sie sind. Würden sich folglich die Tatsachen, die sich in den Gesellschaften abspielen, lediglich durch ihren Grad an Allgemeinheit voneinander unterscheiden, wären es keine, die man als genuine Erscheinungsformen des sozialen Lebens betrachten und zum Gegenstand der Soziologie machen könnte.

Und doch ist die Existenz derartiger Phänomene dermaßen evident, dass sie von Beobachtern angezeigt wurden, die nicht an die Konstitution einer Soziologie dachten. Häufig schon wurde bemerkt, dass eine Menschenmenge, eine Versammlung nicht so empfindet, denkt und handelt wie isolierte Einzelne; dass verschiedenste Gruppierungen - Familie, Korporation, Nation - einen ihnen eigenen "Geist« besitzen, einen Charakter, Gewohnheiten, wie Individuen die ihren. Folglich ist deutlich zu spüren, dass die Gruppe, Menge oder Gesellschaft wirklich eine eigene Natur besitzt, die bei den Individuen eine bestimmte Art des Empfindens, Denkens und Handelns bedingt, und dass diese Individuen nicht dieselben Neigungen, Gewohnheiten noch Vorurteile hätten, hätten sie in anderen Menschengruppen gelebt. Diese Schlussfolgerung kann nun verallgemeinert werden. Zwischen den Ideen, den Handlungen eines isolierten Einzelnen und den kollektiven Manifestationen besteht eine solche Kluft, dass Letztere auf eine neue Natur, auf Kräfte sui generis bezogen werden müssen: andernfalls blieben sie unverständlich.

Nehmen wir beispielhaft die Erscheinungsformen des Wirtschaftslebens der modernen Gesellschaften des Westens: industrielle Warenproduktion, hochgradige Arbeitsteilung, internationaler Handel, Kapitalgesellschaften, Geld, Kredit, Rente, Zinsen, Lohn usw. Man denke nur an die erhebliche Zahl an Grundkenntnissen, Institutionen, Gewohnheiten, die die einfachsten Verrichtungen eines Händlers oder Arbeiters, der seinen Lebensunterhalt zu verdienen sucht, voraussetzen; es ist offensichtlich, dass weder der eine noch der andere die Formen erschafft, die ihre jeweilige Tätigkeit zwangsläufig annimmt: keiner der beiden erfindet den Kredit, die Zinsen, den Lohn, den Warentausch oder das Geld. Was jedem der beiden zugeschrieben werden kann ist die allgemeine Neigung, sich die notwendigen Nahrungsmittel zu beschaffen, sich gegen die Unbill des Wetters zu schützen, oder ist, wenn man will, Unternehmensgeist, Gewinnstreben usw. Selbst dem Anschein nach ganz spontane, natürliche Gefühle wie Gefallen an Arbeit, am Sparen, an Luxus sind in Wirklichkeit Produkt der sozialen Kultur, da sie bei bestimmten Völkern fehlen und zudem selbst innerhalb ein und derselben Gesellschaft je nach Bevölkerungsschicht extrem variieren. Für sich allein würden diese Bedürfnisse zu ihrer Befriedigung eine geringe Zahl an Handlungen erfordern, die auf markante Weise mit den sehr komplexen Formen kontrastiert, in die der Wirtschaftsmensch heute sein Verhalten gießt. Und vom Ursprung außerhalb des Individuums dieser Formen zeugt nicht nur ihre Komplexität, sondern auch und vor allem die Art und Weise, wie sie sich dem Individuum aufzwingen. Dieses ist mehr oder weniger verpflichtet, sich ihnen anzupassen. Teils ist es das Gesetz selbst, das sie zwingt, oder der nicht minder gebieterische Brauch. So musste der Handwerker einst Produkte nach bestimmtem Maß und von bestimmter Qualität anfertigen, und noch heute ist er allen möglichen Reglementierungen unterworfen, kann niemand sich 
weigern, sich mit legalem Geld für seinen legalen Wert bezahlen zu lassen. Mitunter ist es der Lauf der Dinge, an dem das Individuum zerbricht, wenn es sich dagegen auflehnt: So wäre der Händler, der auf einen Kredit verzichten, der Produzent, der seine eigenen Produkte konsumieren wollte, mit einem Wort, der Arbeitende, der für sich allein die Regeln seiner Wirtschaftstätigkeit neu erschaffen wollte, unweigerlich zum Ruin verurteilt.

11 Auch an der Sprache erscheint der soziale Charakter in aller Deutlichkeit: Durch Gebrauch und Unterricht erlernt das Kind eine Sprache, deren Vokabular und Syntax jahrhundertealt sind, deren Ursprünge unbekannt sind, die es folglich als fertige aufnimmt und die es ohne weiterreichende Veränderungen auch weiter so aufnehmen und verwenden soll. Vergeblich wird es versuchen, sich eine eigene Sprache $\mathrm{zu}$ erschaffen: Nicht nur wird es ihm allenfalls gelingen, irgendein vorhandenes Idiom ungeschickt nachzuahmen, diese Sprache wird ihm auch nicht helfen, seine Gedanken angemessen zum Ausdruck zu bringen, es damit zur Isolation und zu einer Art geistigem Tod verurteilen. Der kleinste Verstoß gegen die Regeln und traditionellen Verwendungen stößt auf den heftigen Widerstand der öffentlichen Meinung. Denn eine Sprache ist nicht nur ein System von Wörtern; ihr ist ein besonderer Geist eigen, sie impliziert eine bestimmte Weise des Wahrnehmens, Analysierens und Koordinierens. Folglich zwingt uns die Kollektivität anhand der Sprache die zentralen Formen unseres Denkens auf.

12 Es könnte der Eindruck vorherrschen, dass die ehelichen und häuslichen Beziehungen auf der menschlichen Natur beruhen und dass zu ihrer Erklärung der Verweis auf einige allgemeine, organische und psychologische, Eigenschaften des menschlichen Individuums genügte. Doch zum einen lehrt uns der Blick in die Geschichte, dass die Heirats- und Familientypen äußerst zahlreich und variantenreich waren und noch sind; er erhellt uns die zuweilen außergewöhnliche Kompliziertheit der Heiratsformen und häuslichen Beziehungen. Zum anderen wissen wir alle, dass die häuslichen Beziehungen nicht ausschließlich affektiv sind, dass zwischen uns und möglicherweise unbekannten Verwandten rechtliche Bande vorliegen, die ohne unsere Zustimmung und ohne unser Wissen geknüpft wurden; wir wissen, dass Heirat nicht nur eine Paarung ist, dass Gesetz und Brauchtum dem Mann, der eine Frau ehelicht, bestimmte Handlungen vorschreiben, eine komplizierte Prozedur. Offenkundig vermögen weder die organischen Neigungen des Menschen, sich zu paaren oder fortzupflanzen, noch selbst die Gefühle sexueller Eifersucht oder väterlicher Liebe, die ihm übrigens willkürlich zugestanden werden, auf irgendeinem Niveau die Komplexität und vor allem den verpflichtenden Charakter der ehelichen und häuslichen Sitten und Gebräuche zu erklären.

13 Auch die sehr allgemeinen religiösen Gefühle, die dem Menschen (und selbst den Tieren) gewohnheitsmäßig zugesprochen werden - Achtung und Furcht vor den höheren Wesen, Taumel des Unendlichen - könnten allenfalls sehr einfache und höchst unbestimmte religiöse Akte hervorbringen: Unter der Herrschaft dieser Gefühle würde jeder einzelne Mensch sich die höheren Wesen auf seine Weise vorstellen und ihnen gegenüber die Gefühle bezeugen, die ihm angemessen erscheinen. Eine derartige schlichte, unbestimmte, individuelle Religion hat es jedoch nie gegeben. Der Gläubige glaubt an Dogmen und handelt nach völlig komplizierten Riten, die ihm im Übrigen durch die Kirche, die religiöse Gruppe, der er angehört, vorgegeben werden; im Allgemeinen kennt er diese Dogmen und Riten nur sehr unzulänglich, besteht sein 
religiöses Leben wesentlich aus einer fernen Teilnahme an den Glaubensüberzeugungen und Handlungen von Menschen, die extra dafür bestimmt sind, die sakralen Dinge zu kennen und mit ihnen in Verbindung zu treten; und auch diese Menschen haben ihrerseits die Dogmen und Riten nicht selbst erfunden, sondern haben sie über die Tradition gelernt und wachen nun darüber und schützen sie vor jeder Veränderung. Die individuellen Gefühle keines der Gläubigen erklären mithin weder das komplexe System der eine Religion konstituierenden Vorstellungen und Praktiken noch die Autorität, mit der sich diese vielfache Art des Denkens und Handelns allen Mitgliedern der Kirche aufzwingt.

Die Formen, denen gemäß sich das affektive, geistige, tätige Leben des Individuums entwickelt, existieren folglich vor diesem und überleben es. In seiner Eigenschaft als Mensch isst, denkt, amüsiert er sich usw. Doch wenn er in seinem Handeln durch Neigungen determiniert ist, die allen Menschen gemeinsam sind, dann hängen die genauen Formen, die sein Handeln in jedem historischen Moment annimmt, von ganz anderen Voraussetzungen ab, die von einer Gesellschaft zur anderen variieren und sich innerhalb ein und derselben Gesellschaft im Zeitlauf verändern: das ist die Gesamtheit der kollektiven Gewohnheiten. Unter diesen Gewohnheiten gibt es unterschiedliche Arten. Die einen bedürfen aufgrund ihrer Bedeutung der Reflexion. Man wird sich ihrer bewusst und hält sie in schriftlichen oder mündlichen Formulierungen fest, die zum Ausdruck bringen, wie die Gruppe gewohnheitsmäßig handelt und wie ihre Mitglieder handeln sollen; diese gebieterischen Formeln sind die rechtlichen Regeln, die moralischen Maximen, die rituellen Vorgaben, die Artikel der Dogmen usw. Die anderen bleiben unausgedrückt und diffus, mehr oder minder unbewusst. Dies sind die Gebräuche, die Sitten, die abergläubischen Überzeugungen des Volkes, die befolgt werden, ohne dass man wüsste, dass man an sie gebunden ist noch worin sie genau bestehen. Doch in beiden Fällen ist das Phänomen von gleicher Natur. Es handelt sich immer um Arten des Handelns und Denkens, die von der Tradition ihre Weihe erfahren und durch die Gesellschaft den Individuen aufgezwungen werden. In diesen kollektiven Gewohnheiten und den Transformationen, die sie unaufhörlich durchmachen: darin besteht der eigentliche Gegenstand der Soziologie.

15 Schon jetzt lässt sich übrigens direkt beweisen, dass diese kollektiven Gewohnheiten Manifestationen des Lebens der Gruppe als Gruppe sind. Die vergleichende Geschichte des Rechts und der Religionen hat die Vorstellung eingebürgert, dass bestimmte Institutionen mit bestimmten anderen ein System bilden, dass die einen sich nicht verändern können, ohne dass die anderen sich ebenfalls verändern. Beispielsweise ist bekannt, dass zwischen Totemismus und Exogamie, zwischen der einen oder anderen Praxis und der Organisation des Clans Verbindungen bestehen; man weiß, dass zwischen dem System der patriarchalen Macht und der Regierungsform des Gemeinwesens ein Zusammenhang vorliegt, usw. Allgemein ist es den Historikern zur Gewohnheit geworden, auf die Beziehungen $\mathrm{zu}$ verweisen, von denen die verschiedenen Institutionen ein und derselben Epoche getragen werden, eine Institution nicht von dem Milieu zu trennen, in dem sie aufgetreten ist. Kurzum, man ist immer mehr geneigt, in den Eigenschaften eines sozialen Milieus (Umfang, Dichte, Zusammensetzung usw.) die Erklärung für die allgemeinen Phänomen zu suchen, die sich darin herausbilden. So wird zum Beispiel gezeigt, welche tiefgreifenden Umwälzungen eine landwirtschaftliche Zivilisation durch städtische Ansiedlung erfährt, wie der Wohnsitz die häusliche Organisation bedingt. Wenn nun die Institutionen wechselseitig voneinander abhängen und alle von der Konstitution der 
sozialen Gruppe, dann ist offensichtlich, dass sie Ausdruck von Letzterer sind. Diese Interdependenz der sozialen Phänomene wäre unerklärlich, wenn sie das Produkt einzelner und mehr oder weniger launischer Willensbekundungen wären; sie sind im Gegenteil erklärbar, wenn sie das Ergebnis unpersönlicher und die Individuen selbst beherrschender Kräfte sind.

Ein weiterer Beleg lässt sich aus der Statistik ziehen. Bekanntlich sind die Raten für Heiraten, Geburten, Selbstmorde, Verbrechen in einer Gesellschaft bemerkenswert konstant oder schwanken, wenn überhaupt, nicht abrupt und unregelmäßig und mit hohem Gefälle, sondern im Allgemeinen langsam und geordnet. Ihre Konstanz und Regemäßigkeit gleicht zumindest der jener Phänomene, die wie Sterblichkeit vor allem von physischen Ursachen abhängen. Nun ist es offensichtlich, dass die Gründe, die ein beliebiges Individuum zu einer Heirat oder einem Verbrechen verleiten, vollkommen partikular und zufällig sind; diese Gründe können denn auch nicht die Heirats- oder Verbrechensrate in einer gegebenen Gesellschaft erklären. Es ist folglich von bestimmten sozialen Zuständen auszugehen, die, von rein individuellen Zuständen grundlegend verschieden, die Heiratshäufigkeit und die Kriminalität bedingen. So wäre zum Beispiel nicht verstehbar, warum die Selbstmordrate in mehrheitlich protestantischen Gesellschaften höher liegt als in mehrheitlich katholischen Gesellschaften, im Handelsbereich höher als im landwirtschaftlichen Bereich, wenn man nicht voraussetzte, dass sich in den protestantischen Milieus wie in denen des Handels aufgrund ihrer Organisation eine kollektive Tendenz zum Selbstmord manifestiert.

17 Es gibt mithin genuin soziale Phänomene, die sich von jenen unterscheiden, die die anderen Wissenschaften untersuchen, die wie die Psychologie ebenfalls dem Menschen gewidmet sind. Und genau diese Phänomene bilden den Stoff der Soziologie. Doch ihre Existenz durch eine bestimmte Anzahl von Beispielen und allgemeine Überlegungen zu belegen, ist unzureichend. Man möchte gern noch wissen, durch welche Anzeichen sie zu erkennen sind, um sowohl der Gefahr zu entgehen, sie zu übersehen, als auch, sie mit Phänomenen $\mathrm{zu}$ verwechseln, die in den Zuständigkeitsbereich der anderen Wissenschaften gehören. Nach dem bisher Gesagten zeichnet sich die Natur des Sozialen dadurch aus, dass sie zur Natur des Individuellen gleichsam hinzukommt; sie äußert sich durch Ideen oder Handlungen, die, obwohl wir zu ihrem Auftreten beitragen, uns völlig von außen aufgezwungen werden. Es geht genau darum, dieses Zeichen der Äußerlichkeit zu entdecken.

In einer großen Zahl von Fällen ist der verpflichtende Charakter, der soziale Weisen des Handelns und Denkens kennzeichnet, das beste Kriterium, das man sich wünschen kann. Tief in unserem Innern eingebrannt oder in rechtlichen Formeln zum Ausdruck gebracht, existiert eine Vielzahl strikt obligatorischer Regeln juridischer, religiöser und moralischer Natur, denen spontan gehorcht wird oder die qua Zwang befolgt werden. Die meisten Individuen gehorchen ihnen; selbst wer sie verletzt, weiß, dass er sich einer Verpflichtung entzieht; auf alle Fälle erinnert ihn die Gesellschaft an den verpflichtenden Charakter ihrer Ordnung und belegt ihn mit einer Strafe. Worin die Beschaffenheit und Intensität der Strafe auch bestehen mag - Exkommunikation oder Tod, Schmerzensgeld oder Gefängnis, öffentliche Verachtung, Schande, bloße Feststellung von Exzentrizität -, das Phänomen bleibt in den verschiedenen Graden und unterschiedlichen Formen immer dasselbe: Die Gruppe protestiert gegen die Verletzung der kollektiven Regeln des Denkens und Handelns. Und die Bedeutung 
dieses Protests kann nur sein: die von der Gruppe erzwungenen Weisen des Denkens und Handelns sind die ihr ureigensten. Dass sie Verstöße dagegen nicht toleriert, ist darin begründet, dass sie in diesen Weisen Erscheinungsformen ihrer Persönlichkeit sieht, und indem man gegen sie verstößt, schmälert man sie, zerstört man sie. Und außerdem: wenn die Regeln des Denkens und Handelns nicht sozialen Ursprungs wären, woher sollten sie kommen? Eine Regel, der sich ein Individuum unterworfen fühlt, kann nicht das Werk dieses Individuums sein: denn jede Verpflichtung impliziert eine gegenüber dem verpflichteten Subjekt höhere Autorität, die ihm Respekt einflößt, den wesentlichem Bestandteil des Gefühls von Verpflichtung. Wenn also das Eingreifen übernatürlicher Wesen ausgeschlossen wird, ist außerhalb des Individuums und über ihm nur eine Quelle der Verpflichtung auffindbar: die Gesellschaft oder vielmehr das Gesamt der Gesellschaften, dessen Mitglied es ist.

Hier liegt also eine Gesamtheit leicht erkennbarer und bedeutsamster sozialer Phänomene vor. Denn Recht, Moral, Religion machen einen beträchtlichen Teil des sozialen Lebens aus. Selbst in den niederen Gesellschaften gibt es kaum kollektive Manifestationen, die nicht zu einer dieser Kategorien gehören. Der Mensch hat dort gewissermaßen weder ein eigenes Denken noch Handeln; Sprechen, die wirtschaftlichen Operationen, selbst die Kleidung gewinnen dort oft einen religiösen und folglich verpflichtenden Charakter. In den höheren Gesellschaften dagegen wird in vielen Fällen der soziale Druck nicht in der ausdrücklichen Form der Verpflichtung spürbar: In Fragen der Wirtschaft, des Rechts, selbst der Religion scheint das Individuum weitgehend autonom zu sein.

Das heißt nicht, dass aller Zwang abwesend wäre: Weiter vorn haben wir ausgeführt, unter welchen Aspekten er sich im wirtschaftlichen und sprachlichen Bereich äußert, und wie viel es bedurfte, damit der Einzelne in diesen Hinsichten frei war, nach Gutdünken zu handeln. Es gibt jedoch keine offen erklärte Verpflichtung, keine festgelegten Sanktionen; Innovation, Verstoß sind nicht prinzipiell verboten. Deshalb muss ein anderes Kriterium gesucht werden, das erlaubt, jene Gewohnheiten herauszuheben, deren besondere Natur nicht minder unbestreitbar, aber nicht auf Anhieb sichtbar ist.

Unbestreitbar ist sie in der Tat, weil jedes Individuum diese Gewohnheiten bereits geformt und gleichsam instituiert vorfindet; weil es nicht deren Erschaffer ist, weil sie ihm von außen gegeben sind, sie also präetabliert sind. Ob es dem Individuum verboten ist oder nicht, sich von ihnen abzusetzen: sie sind bereits da, wenn es sich fragt, wie es handeln soll; sie geben ihm Modelle des Verhaltens vor. Man sieht also gewissermaßen, wie sie in einem bestimmten Moment von außen in es eindringen. In den meisten Fällen erfolgt dieses Eindringen durch eine allgemeine oder besondere Erziehung. So erhält jede Generation von der ihr vorangegangenen die moralischen Vorschriften, die üblichen Höflichkeitsregeln, ihre Sprache, ihre grundlegenden Geschmacksneigungen, genauso wie jeder Arbeitende von seinen Vorgängern die Regeln seiner Berufstechnik vermittelt bekommt. Erziehung ist die Operation, mit der dem individuellen Wesen in jedem von uns ein soziales Wesen, dem animalischen Wesen ein moralisches Wesen hinzugefügt wird; sie ist das Verfahren, dank dem das Kind rasch sozialisiert wird. Diese Beobachtungen liefern uns eine Charakteristik der sozialen Tatsache, die die vorhergehende an Allgemeinheit übertrifft: sozial sind all die Weisen des Handelns und Denkens, die das Individuum als bereits bestehende vorfindet und deren Weitergabe weitgehend über die Erziehung erfolgt. 

- und Institution scheint tatsächlich das angemessenste. Denn was ist eine Institution, wenn nicht eine bereits bestehende Gesamtheit von Handlungen und Vorstellungen, die die Individuen vorfinden und die sich ihnen mehr oder weniger aufzwingen? Es gibt keinen Grund, diesen Ausdruck ausschließlich, wie es gewöhnlich geschieht, den grundlegenden sozialen Übereinkünften vorzubehalten. Wir verstehen unter diesem Wort also die Gebräuche und Moden, die Vorurteile und die abergläubischen Überzeugungen ebenso wie die politischen Verfassungen und zentralen rechtlichen Organisationen; denn alle diese Phänomene sind von gleicher Natur und nur graduell verschieden. Die Institution ist, kurz gesagt, innerhalb der sozialen Ordnung, was die Funktion in der biologischen Ordnung ist: Und wie die Wissenschaft vom Leben die Wissenschaft von den Lebensfunktionen ist, so ist die Wissenschaft von der Gesellschaft die Wissenschaft von den in diesem Sinne definierten Institutionen.

Aber die Institution, wird man einwenden, ist doch Vergangenheit; ist, definitionsgemäß, das Festgelegte, nicht das Lebendige. In jedem Augenblick ergibt sich in den Gesellschaften Neues, von den täglichen Variationen der Mode bis zu den großen Revolutionen in Politik und Moral. Doch alle diese Wechselerscheinungen sind, auf unterschiedlichen Stufen, stets Veränderungen existierender Institutionen. Revolutionen bestanden noch nie in der plötzlichen Ersetzung der ganzen bestehenden Ordnung durch eine neue Ordnung; sie sind und können nie anderes sein als mehr oder weniger rasche, mehr oder weniger umfassende Umwandlungen. Nichts kommt von nichts: Die neuen Institutionen können nur mit den alten gemacht werden, denn diese allein existieren. Und damit unsere Definition alles Definierte umfasst, reicht es folglich, dass wir uns nicht an eine enge statische Formel halten, die Soziologie nicht auf die Untersuchung angeblich starrer Institutionen beschränken. Denn eine derart begriffene Institution ist nur eine Abstraktion. Wirkliche Institutionen leben, das heißt, wandeln sich fortwährend: Die Regeln des Handelns werden nicht in jedem aufeinanderfolgenden Moment auf die gleiche Weise weder verstanden noch angewandt, wohingegen die Formeln, anhand deren sie zum Ausdruck kommen, buchstäblich dieselben bleiben. Die lebendigen Institutionen, so wie sie sich bilden, funktionieren und umbilden, stellen folglich die genuin sozialen Phänomene, die Gegenstände der Soziologie, dar.

24

Die einzigen Tatsachen, die man nicht ohne Grund als soziale ansehen kann und die sich doch nur schwer in der Definition der Institutionen unterbringen ließen, sind jene, die in Gesellschaften ohne Institutionen auftreten. Doch die einzigen institutionenlosen Gesellschaften sind entweder instabile und vergängliche soziale Aggregate wie die Massen oder solche, die gerade im Entstehen sind. Von den einen wie den anderen kann man sagen, dass sie noch keine Gesellschaften im eigentlichen Sinne sind, sondern nur Gesellschaften im Werden, mit dem Unterschied, dass die einen ihrer Bestimmung nach bis ans Ende ihrer Entwicklung gehen, ihre soziale Natur realisieren, während die anderen verschwinden, bevor es ihnen gelungen ist, sich definitiv auszubilden. Wir befinden uns hier mithin an den Scheidelinien zwischen dem Reich des Sozialen und Reichen niederer Art. Die fraglichen Phänomene sind eher auf dem Weg, soziale zu werden, als bereits soziale zu sein. Es ist daher nicht verwunderlich, dass sie in keinen Rahmen irgendeiner Wissenschaft wirklich passen. Sicher darf die Soziologie nicht von ihnen absehen, aber sie stellen nicht ihren eigentlichen Gegenstand dar. Im Übrigen haben wir mit der vorangehenden Analyse keineswegs

Trivium, 32 | 2021 
eine endgültige und umfassende Definition aller sozialen Phänomene zu entdecken gesucht. Es genügt, aufgezeigt zu haben, dass es derart zu Recht bezeichnete Tatsachen gibt, und auf einige Zeichen hingewiesen zu haben, an denen die wichtigsten unter ihnen erkennbar sind. Die Zukunft wird ganz sicher an die Stelle dieser Kriterien weniger mangelhafte setzen.

\section{Von der soziologischen Erklärung}

So verfügt die Soziologie über einen eigenen Gegenstand, da es genuine soziale Tatsachen gibt. Uns bleibt zu prüfen, ob sie die zweite von uns erwähnte Voraussetzung erfüllt, das heißt, ob es einen Modus der soziologischen Erklärung gibt, der in keinem anderen aufgeht. Der erste auf diese Tatbestände methodisch angewandte Erklärungsmodus ist jener, der lange Zeit über in der sogenannten Geschichtsphilosophie in Gebrauch war. Geschichtsphilosophie ist in der Tat die der eigentlichen Soziologie unmittelbar vorangehende Form soziologischer Spekulation gewesen. Die Soziologie ist aus der Geschichtsphilosophie erwachsen: Comte ist der direkte Nachfolger von Condorcet, und er selbst hat eher eine Geschichtsphilosophie entwickelt als soziologische Entdeckungen gemacht. Das Kennzeichen philosophischer Erklärung ist, dass sie voraussetzt, dem Menschen, der Menschheit allgemein sei qua seiner bzw. ihrer Natur eine bestimmte Entwicklung vorbestimmt, deren Richtung man durch eine summarische Untersuchung der historischen Fakten zu entdecken sucht. Aus Prinzip und Methode vernachlässigt man also die Einzelheiten und hält sich an die gröbsten Linien. Man sucht nicht zu erklären, warum man in dieser bestimmten Art von Gesellschaft, zu diesem Zeitabschnitt ihrer Entwicklung auf diese oder jene Institution trifft; gesucht wird lediglich, auf welches Ziel hin sich die Menschheit entwickelt, markiert werden die Etappen, die notwendig waren, um sich dem Ziel zu nähern.

Das Ungenügen einer solchen Erklärung muss nicht eigens bewiesen werden. Nicht nur lässt sie willkürlich den hauptsächlichen Teil der Realität außer Acht; sondern alle diese Denksysteme erweisen sich auch als grundlos, da sich heutzutage nicht mehr vertreten lässt, dass die Menschheit einem einzigen Weg folgt und sich in eine einzige Richtung hin entwickelt. Und doch findet man auch heute noch in gewissen soziologischen Doktrinen derartige Erklärungsmuster, die sich allenfalls dem Anschein nach von den vorhergehenden unterscheiden. Unter dem Vorwand, dass eine Gesellschaft lediglich aus individuellen Einzelnen besteht, werden die bestimmenden Ursachen, mit denen soziale Tatsachen erklärt werden sollen, denn auch in der Natur des Individuums gesucht. Spencer und Tarde etwa gehen auf diese Weise vor. Spencer hat nahezu den gesamten ersten Band seiner Soziologie der Untersuchung des primitiven Menschen auf physischer, emotionaler und intellektueller Ebene gewidmet; anhand der Eigenschaften der Natur dieser Primitiven erklärt er die bei den ältesten oder den ursprünglichsten Völkern beobachteten Institutionen, Institutionen, die sich dann im Verlauf der Geschichte entsprechend sehr allgemeinen Evolutionsgesetzen umgestalten. Tarde sieht in den Gesetzen der Nachahmung die obersten Prinzipien der Soziologie: Die sozialen Phänomene stellen zumeist nützliche Handlungsweisen dar, die von bestimmten Einzelnen erfunden wurden und dann von den anderen nachgeahmt werden. Dasselbe Erklärungsmuster findet sich in bestimmten Spezialwissenschaften wieder, die soziologische sind oder sein sollten. So finden die klassischen Wirtschaftswissenschaftler in der individuellen Natur des homo œeconomicus die 
Prinzipien einer hinreichenden Erklärung aller wirtschaftlichen Tatsachen: Da der Mensch immer unter kleinstmöglichem Aufwand den größten Nutzen sucht, müssen die ökonomischen Beziehungen notwendigerweise so und so aussehen. Ähnlich suchen die Theoretiker des Naturrechts nach den rechtlichen und moralischen Merkmalen der menschlichen Natur, und die Rechtsinstitutionen bilden in ihren Augen die mehr oder weniger erfolgreichen Versuche, der Strenge dieser Natur zu genügen; der Mensch wird sich nach und nach seiner bewusst, und die positiven Rechte sind die annähernden Realisierungen des Rechts, das er in sich trägt.

Das Ungenügen dieser Lösungen wird deutlich, sobald man erkennt, dass es soziale Tatsachen, soziale Realitäten gibt, das heißt, sobald man den eigentlichen Gegenstand der Soziologie ausgemacht hat.

Wenn die sozialen Phänomene tatsächlich die Erscheinungsformen des Lebens der Gruppen als solche bilden, sind sie viel zu komplex, als dass Betrachtungen zur menschlichen Natur im Allgemeinen darüber Aufschluss geben könnten. Nehmen wir erneut als Beispiel die Institutionen Heirat und Familie. Die Beziehungen zwischen den Geschlechtern sind recht komplizierten Regeln unterworfen: Die Familienorganisation ist in ein und derselben Gesellschaft sehr stabil, variiert aber erheblich von einer Gesellschaft zur anderen; zudem ist sie eng an die politische und ökonomische Organisation gebunden, die in verschiedenen Gesellschaften ebenfalls typische Unterschiede aufweisen. Wenn dies soziale Phänomene sind, die es zu erklären gilt, stellen sich einige konkrete Probleme: Wie haben sich die verschiedenen ehelichen und häuslichen Systeme ausgebildet? Lassen sie sich aufeinander beziehen, spätere von früheren Formen unterscheiden, wobei jene als Produkt der Transformation von diesen erscheinen? Wenn dies möglich ist, wie lassen sich diese Transformationen erklären, welches sind ihre Voraussetzungen? Wie wirken sich die Formationen der Familienorganisation auf die politischen und ökonomischen Organisationen aus? Zum anderen: Ist ein bestimmtes häusliches Regime einmal ausgebildet, wie funktioniert es? Auf diese Fragen können Soziologen, die als Prinzip ihrer Erklärungen lediglich auf die Individualpsychologie zurückgreifen, keine Antworten liefern. Denn sie können von diesen derart vielfältigen und variablen Institutionen nur Aufschluss geben, indem sie sie auf sehr allgemeine Elemente der organisch-psychischen Konstitution des Einzelmenschen beziehen: Geschlechtsinstinkt, Neigung $\mathrm{zu}$ alleinigem und eifersüchtigem Besitz einer einzigen weiblichen Person, Mutter- und Vaterliebe, Abscheu vor Geschlechtsverkehr zwischen Blutsverwandten usw. Aber derartige Erläuterungen sind schon von einem rein philosophischen Standpunkt aus suspekt: Sie bestehen einfach darin, dem Menschen die Gefühle zuzuschreiben, die sein Verhalten offenbart, während gerade diese Gefühle zu erklären wären; was kurzum darauf hinausläuft, das Phänomen durch die geheimen Kräfte der Substanzen, die Flamme durch das Phlogiston und den Fall der Körper durch ihre Schwerkraft zu erklären. Außerdem unterlassen sie es, zwischen den Phänomenen genaue Beziehungen der Koexistenz und der Aufeinanderfolge festzulegen, isolieren sie vielmehr willkürlich und stellen sie außerhalb von Zeit und Raum dar, losgelöst von jedem bestimmten Milieu. Selbst wenn man als eine Erklärung der Monogamie die These ansehen würde, dass dieses Eheregime besser als ein anderes die menschlichen Instinkte befriedigte oder besser als jedes andere die Freiheit und Würde der beiden Eheleute vereinte, wäre noch zu eruieren, warum dieses Regime eher in dieser als in jener Gesellschaft, in diesem und nicht jenem Moment in der Entwicklung einer Gesellschaft auftritt. Drittens sind die wesentlichen Eigenschaften der menschlichen Natur bis auf kleinere 
Abstufungen und Nuancen überall gleich. Wie könnten sie derart vielfältige Formen, die jede Institution nacheinander annimmt, explizieren? Vater- und Mutterliebe, die zärtlichen Gefühle für Kinder sind offensichtlich bei den Primitiven wie den Zivilisierten identisch; doch welche Kluft besteht zwischen der primitiven Organisation der Familie und ihrer heutigen Verfassung, und welche Veränderungen haben sich zwischen diesen Extremen nicht ereignet! Letztlich lassen sich die so präzisen und komplexen Formen, unter denen sich die historischen Realitäten immer zeigen, nicht anhand unbestimmter Neigungen des Menschen entschlüsseln. Der Egoismus, der den Menschen dazu verleiten kann, sich nützliche Dinge anzueignen, ist nicht die Quelle solcher komplizierten Regeln, die in jeder geschichtlichen Epoche das Eigentumsrecht ausmachen, Regeln im Hinblick auf Grund und Boden, Nutznießung, beweglichen und unbeweglichen Besitz, Dienstleistungen usw. Und dennoch gibt es das Eigentumsrecht in abstracto nicht. Was es gibt, ist das Eigentumsrecht, wie es im zeitgenössischen Frankreich oder im antiken Rom organisiert ist bzw. war, samt der ihm zugrunde liegenden vielfältigen Prinzipien. Die so verstandene Soziologie kann auf diese Weise nur zu gänzlich allgemeinen und aufgrund ihrer Unbestimmtheit nahezu unfassbaren Grundzügen der Institutionen gelangen. Übernimmt man diese Prinzipien, muss man eingestehen, dass der größte Teil der sozialen Realität (die Institutionen in all ihren Einzelheiten) unerklärt und unerklärbar bleibt. Natürlich und verstehbar wären nur die Phänomene, die durch die im tiefsten Innern immer identische Menschennatur im Allgemeinen bestimmt sind; alle besonderen Merkmale, durch die die Institutionen je nach Zeit und Ort ihren spezifischen Charakter gewinnen, alles, was die sozialen Individualitäten unterscheidet, gilt als künstlich und akzidentiell; man sieht darin entweder die Resultate zufälliger Erfindungen oder die Produkte der individuellen Akte von Gesetzgebern, von Mächtigen, die die Gesellschaften absichtlich auf die von ihnen undeutlich wahrgenommenen Ziele hin führen. Man wird damit dazu verleitet, aus der Wissenschaft alle genau bestimmten Institutionen als nicht verstehbar auszustoßen, das heißt die sozialen Tatsachen selbst, die genuinen Gegenstände der soziologischen Wissenschaft. Anders gesagt, man vernichtet auf diese Weise mit dem definierten Objekt einer Sozialwissenschaft gleich die Sozialwissenschaft selbst und begnügt sich damit, bei der Philosophie und der Psychologie nach ganz allgemeinen Hinweisen zu den Schicksalen des in der Gesellschaft lebenden Menschen zu fragen.

Diesen durch äußerste Allgemeinheit gekennzeichneten Erklärungen stehen jene gegenüber, die man eigentliche historische Erklärungen nennen könnte. Das heißt nicht, dass es historisch nicht auch andere gegeben hätte, aber die uns jetzt beschäftigenden finden sich ausschließlich bei den Historikern. Aufgrund der besonderen Bedingungen seiner Arbeit dazu verpflichtet, sich nur mit einer bestimmten Gesellschaft in einer bestimmten Epoche zu beschäftigen, vertraut mit dem Geist, der Sprache, den besonderen Charakterzügen dieser Gesellschaft und dieser Epoche, weist der Historiker die natürliche Tendenz auf, nur das in den Tatsachen zu sehen, was sie von den anderen unterscheidet, was ihnen in jedem Einzelfall eine ihnen eigene Physiognomie verleiht, in einem Wort: was sie unvergleichbar macht. Im Versuch, die Mentalität der Völker wiederzufinden, deren Geschichte er studiert, neigt er dazu, diejenigen als verständnislos und inkompetent zu verurteilen, die nicht wie er im intimen Umgang mit diesen Völkern gelebt haben. Folgerichtig misstraut er tendenziell jedem Vergleich, jeder Verallgemeinerung. Untersucht er eine Institution, richtet sich seine Aufmerksamkeit auf die individuellsten Merkmale, jene, die den besonderen Umständen geschuldet sind, unter denen sie entstanden ist oder sich 
verändert hat; sie erscheint ihm daher als nicht zu trennen von diesen Umständen. So soll die patriarchale Familie eine wesentlich römische Angelegenheit gewesen sein, Feudalismus eine besondere Institution unserer mittelalterlichen Gesellschaften usw. Aus dieser Perspektive können die Institutionen nur als zufällige und lokale Kombinationen in den Blick geraten, die von gleichermaßen zufälligen und lokalen Bedingungen abhängen. Während die Philosophen und Psychologen uns Theorien vorsetzten, die gewissermaßen für die gesamte Menschheit gelten, lassen sich die für Historiker einzig möglichen Erklärungen lediglich auf eine bestimmte, in einem präzisen Moment ihrer Entwicklung betrachtete Gesellschaft anwenden. Abgelehnt werden überall wirksame allgemeine Ursachen, deren Erforschung sinnvoll wäre. Aufgabe ist es, ein partikulares Ereignis nach dem anderen aneinanderzureihen. In Wirklichkeit setzt man in den Tatsachen eine unendliche Diversität und eine unendliche Kontingenz voraus.

Dieser im engen Sinn historischen Methode der Erklärung sozialer Tatsachen sind zunächst die durch die vergleichende Methode gewonnenen Befunde entgegenzusetzen: Bereits jetzt hat die vergleichende Geschichte der Religionen, des Rechts und der Sitten das Vorhandensein unbestritten identischer Institutionen bei den verschiedensten Völkern aufgewiesen; diesen Übereinstimmungen die Nachahmung einer Gesellschaft durch andere erklärend zugrunde $\mathrm{zu}$ legen ist undenkbar; zugleich lassen sie sich unmöglich als zufällige betrachten: Es kann nicht sein, dass ähnliche Institutionen in einer beliebigen wilden Völkerschaft auf lokale und zufällige Ursachen zurückgehen und in einer bestimmten zivilisierten Gesellschaft auf andere, aber gleichermaßen lokale und zufällige Ursachen. Zum anderen stellen die fraglichen Institutionen nicht nur sehr allgemeine Praxisformen dar, von denen man behaupten könnte, dass sie von Menschen unter gleichen Umständen ganz natürlich erfunden worden seien. Es handelt sich nicht nur um bedeutsame Mythen wie die Sintflut, Riten wie die Opferung, häusliche Organisationen wie die mütterliche Familie, rechtliche Praktiken wie die Blutrache; es handelt sich im Weiteren um höchst komplexe Legenden, abergläubische Überzeugungen, ganz besondere Bräuche wie die Couvade oder die Leviratsehe. Hat man derartige Ähnlichkeiten festgestellt, wird es untragbar, die vergleichbaren Phänomene anhand von Ursachen zu erklären, die für eine Gesellschaft oder Epoche spezifisch sind: Der Geist sperrt sich, Regelmäßigkeit und Ähnlichkeit als zufällig zu erachten.

31 Richtig ist, dass die Geschichte zwar nicht aufweist, aus welchen Gründen es analoge Institutionen in ähnlichen Zivilisationen gibt, sie aber beansprucht, die Tatsachen dadurch zu erklären, dass sie sie chronologisch aneinanderreiht und im Einzelnen die Umstände beschreibt, unter denen sich ein historisches Ereignis ereignet hat. Doch derartigen Beziehungen reiner Aufeinanderfolge gebricht es an Notwendigkeit wie Verstehbarkeit. Denn die Art, wie Historiker einem Ereignis ein anderes Ereignis als dessen Ursache zuweisen, ist vollkommen arbiträr, mitnichten methodisch und folglich völlig irrational. Denn induktive Verfahren sind nur dort anwendbar, wo Vergleiche leicht sind. Sobald sie beanspruchen, eine einzelne Tatsache durch eine andere einzelne Tatsache zu erklären, sobald sie in Abrede stellen, dass zwischen den Tatsachen notwendige und konstante Zusammenhänge bestehen, können Historiker Ursachen gar nicht anders als durch unmittelbare Intuition wahrnehmen, ein Verfahren, das sich jeder Regulierung wie Kontrolle entzieht. Daraus folgt, dass die historische Erklärung nicht nur unfähig ist, die beobachteten Ähnlichkeiten begreiflich zu machen, sondern nicht einmal über ein singuläres Ereignis Aufschluss zu geben vermag; sie bietet dem 
Verständnis lediglich Phänomene an, die, weil als singulär, zufällig und willkürlich aneinandergereiht begriffen, nicht verständlich sind.

Ganz anders die eigentliche soziologische Erklärung, so wie sie begriffen werden muss, wenn man die von uns vorgeschlagene Definition des sozialen Phänomens akzeptiert. Zum einen gibt sie nicht nur als Aufgabe vor, bis zu den allgemeinsten Aspekten des sozialen Lebens vorzudringen. Zwischen den sozialen Tatsachen je nach Grad an Allgemeinheit zu unterscheiden ist unstatthaft: die allgemeinste ist so natürlich wie die partikularste, beide sind gleichermaßen explizierbar. So können und müssen alle Tatsachen, die alle Merkmale wie die der sozialen Tatsache aufweisen, Gegenstand der Forschung sein. Es kann vorkommen, dass es welche gibt, die der Soziologe aktuell nicht in ein System einfügen kann; aber es gibt keine, die er rechtens, a priori, aus der Wissenschaft und der Erklärung ausschließen darf. Die so verstandene Soziologie stellt damit keinen allgemeinen und fernen Überblick über die kollektive Realität dar, sondern deren Analyse, die so tiefgehend und so umfassend wie möglich ist. Sie verpflichtet sich in der Untersuchung des Details zu ebenso großer Genauigkeit wie der Historiker. Keinen noch so winzigen Tatbestand darf sie vernachlässigen, weil er angeblich ohne wissenschaftliches Interesse ist. Schon jetzt lassen sich welche nennen, die von geringer Bedeutung schienen und doch symptomatisch sind für wesentliche soziale Zustände, zu deren Verständnis sie helfen können. Die Erbfolge etwa steht in engem Zusammenhang mit der Konstitution der Familie; und ob die Aufteilung nach Stammfolge oder Kopfzahl erfolgt, ist nicht zufällig, beide Aufteilungsformen entsprechen sehr unterschiedlichen Arten von Familie. Ähnlich ist auch der Strafvollzug für den interessant, der den Zustand der öffentlichen Meinung zur Frage der Strafe in der betreffenden Gesellschaft untersuchen will.

Zum anderen, während die Historiker die Tatsachen beschreiben, sie aber nicht im eigentlichen Sinne erklären, versucht die Soziologie eine der Vernunft genügende Erklärung zu liefern. Sie sucht zwischen den Tatsachen nicht bloße Beziehungen der Aufeinanderfolge zu finden, sondern verstehbare Beziehungen. Sie will zeigen, wie die sozialen Tatsachen hervorgebracht wurden, Ergebnis welcher Kräfte diese sind. Sie muss folglich die Tatsachen erklären, so wie sie durch ihre bestimmenden Ursachen, ihre nächsten und unmittelbaren Ursachen, definiert sind, die in der Lage sind, sie hervorzubringen. Sie begnügt sich in der Folge nicht damit, wie gewisse Soziologen, auf sehr allgemeine und entfernte Ursachen hinzuweisen, die jedenfalls unzureichend sind und nicht in direktem Zusammenhang mit den Tatsachen stehen. Da die sozialen Tatsachen spezifisch sind, lassen sie sich nur durch gleichartige Ursachen erklären. Die soziologische Erklärung verfährt also derart, dass sie von einem sozialen Phänomen zum nächsten geht. Einen Zusammenhang stellt sie nur zwischen sozialen Phänomenen her. Auf diese Weise zeigt sie, wie Institutionen sich wechselseitig erzeugen; zum Beispiel, wie sich der Ahnenkult auf der Grundlage der Bestattungsriten entwickelte. In anderen Fällen entdeckt sie regelrechte Verwachsungen sozialer Phänomene: Zum Beispiel wird der weitverbreitete Begriff des Gottesopfers durch eine Art Verschmelzung zwischen bestimmten Opferriten und bestimmten mythischen Vorstellungen expliziert. Manchmal kommt es zur Verkettung von Tatbeständen der sozialen Struktur, etwa wenn die Bildung von Städten mit mehr oder weniger umfänglichen Migrationsbewegungen von Dörfern in die Städte, von ländlichen Bezirken in Industriebezirke, oder mit Kolonisierungsbewegungen, dem Zustand der Kommunikation usw. zusammengebracht werden können. Oder bestimmte Institutionen werden durch die Struktur von Gesellschaften eines bestimmten Typs 
erläutert, zum Beispiel bringt das Zusammensiedeln in Städten bestimmte Ausprägungen des Eigentums, des Kultus usw. hervor.

Aber wie bringen sich die sozialen Tatsachen wechselseitig hervor? Wenn wir sagen, dass Institutionen auf dem Weg der Entwicklung, der Verwachsung usw. Institutionen hervorbringen, heißt das nicht, dass wir sie als eine Art autonomer Realitäten auffassen, die aus sich heraus eine ganz besondere geheimnisvolle Wirksamkeit entfalten könnten. Und auch wenn wir diese oder jene soziale Praxis mit einer bestimmten Form der Gruppe verknüpfen, bedeutet das nicht, dass wir es als möglich erachten, dass die geografische Verteilung der Individuen das soziale Leben direkt und ohne Vermittlung affiziert. Die Institutionen existieren ausschließlich in den Repräsentationen, die sich die Gesellschaft von ihnen macht. Ihre gesamte lebendige Kraft erwächst aus den Gefühlen, die sich auf sie richten; sind sie stark und respektiert, dann deshalb, weil diese Gefühle lebendig sind; schwinden sie, dann weil sie jede Autorität in den Bewusstseinen verloren haben. Und wenn Veränderungen der sozialen Struktur sich auf die Institutionen auswirken, dann weil sie die Verfassung der Ideen und die Neigungen, deren Objekt sie sind, modifizieren. Wenn zum Beispiel die Bildung des städtischen Gemeinwesens das System der patriarchalen Familie sehr verstärkt, dann weil dieser Komplex an Ideen und Gefühlen, aus dem das Familienleben besteht, sich notwendig in dem Maße verwandelt, wie das Gemeinwesen an Dichte gewinnt. Um den üblichen Sprachgebrauch zu verwenden, könnte man sagen, dass die sozialen Tatsachen ihre ganze Kraft aus der öffentlichen Meinung gewinnen. Die öffentliche Meinung diktiert die moralischen Regeln und sanktioniert sie direkt oder indirekt. Man könnte sogar so weit gehen und behaupten, dass jegliche Veränderung in den Institutionen im Grunde eine Veränderung in der öffentlichen Meinung ist: Weil die kollektiven Gefühle des Mitleids mit dem Straftäter den kollektiven Gefühlen widerstreiten, die Bestrafung verlangen, nimmt der Strafvollzug fortschreitend mildere Formen an. Alles vollzieht sich in der Sphäre der öffentlichen Meinung, aber diese ist im genuinen Sinne das, was wir als System der kollektiven Vorstellungen bezeichnen. Die sozialen Tatsachen sind folglich Ursachen, weil sie Vorstellungen sind oder auf Vorstellungen einwirken. Der innere Fundus des sozialen Lebens ist ein Ensemble von Vorstellungen.

In diesem Sinne könnte man also sagen, dass die Soziologie eine Psychologie ist. Wir würden diese Formulierung akzeptieren, unter der ausdrücklichen Voraussetzung eines Zusatzes, wonach diese Psychologie sich in spezifischer Hinsicht von der Individualpsychologie unterscheidet. Die Vorstellungen, mit denen beide sich beschäftigen, sind ihrer jeweiligen Beschaffenheit nach grundsätzlich verschieden. Das ergab sich bereits aus unseren Ausführungen $\mathrm{zu}$ den Merkmalen des sozialen Phänomens, denn es ist offensichtlich, dass Tatsachen mit derart unterschiedlichen Eigenschaften nicht von derselben Art sein können. Im Bewusstsein der Individuen gibt es von den individuellen Vorstellungen unterschiedene kollektive Vorstellungen. Zweifellos bestehen Gesellschaften nur aus Individuen, und folglich gehen kollektive Vorstellungen auf die Art und Weise zurück, wie individuelle Bewusstseine innerhalb einer bestehenden Gruppe agieren und reagieren können. Doch diese Aktionen und Reaktionen entbinden neuartige psychische Phänomene, die in der Lage sind, sich aus sich selbst heraus $\mathrm{zu}$ entwickeln, sich wechselseitig $\mathrm{zu}$ verändern, und deren Gesamtheit ein definiertes System bildet. Nicht nur bestehen kollektive Vorstellungen aus anderen Elementen als individuellen Vorstellungen, auch ihr objekt ist in Wirklichkeit ein jeweils anderes. Denn was sie zum Ausdruck bringen, ist der Zustand 
der Gesellschaft selbst. Während die Bewusstseinstatsachen der Individuen immer auf eine mehr oder weniger entfernte Weise einen Zustand des Organismus zum Ausdruck bringen, äußert sich in den kollektiven Vorstellungen in einem bestimmten Grad immer ein Zustand der sozialen Gruppe: Sie übertragen (oder um den philosophischen Sprachgebrauch zu verwenden: sie »symbolisieren«) deren aktuelle Struktur, die Art und Weise, wie sie auf dieses oder jenes Ereignis reagiert, das Gefühl, dass sie von sich selbst oder von ihren ureigenen Interessen hat. Das psychische Leben der Gesellschaft besteht also aus einem ganz anderen Stoff als das der Individuen.

Das heißt nun nicht, dass zwischen den beiden jede Kontinuität aufgelöst wäre. Sicher sind die Bewusstseine, aus denen die Gesellschaft gebildet ist, neuartig kombiniert, woraus sich die neuen Realitäten ergeben. Dennoch kann anhand einer Serie von Übergängen durchaus von Tatsachen des individuellen Bewusstseins zu kollektiven Vorstellungen übergegangen werden. Einige dieser Vermittlungsglieder sind leicht auszumachen: Vom Individuellen geht man unmerklich zur Gesellschaft über, wenn man zum Beispiel die Tatbestände der epidemischen Nachahmung, der Massenbewegungen, der kollektiven Halluzination usw. seriell aneinanderreiht. Umgekehrt wird das Soziale wieder individuell. Es existiert nur in den individuellen Bewusstseinen, aber jedes Bewusstsein erfasst nur eine Parzelle. Zudem noch wird der Eindruck der sozialen Dinge durch den besonderen Zustand des Bewusstseins verändert, das sie aufnimmt. Jeder spricht auf seine Art seine Muttersprache, jeder Autor legt sich am Ende seine Syntax, seinen Lieblingswortschatz zu. Ähnlich erstellt sich jeder Einzelne seine Moral, hat er seine individuellen Moralvorstellungen. Ähnlich betet und verehrt jeder nach seinen Neigungen. Doch diese Tatsachen sind nicht erklärbar, wenn man sich zu ihrem Verständnis ausschließlich auf individuelle Phänomene beruft; erklärbar sind sie vielmehr, wenn man von den sozialen Tatsachen ausgeht. Nehmen wir zum Beleg einen konkreten Fall individueller Religion, den des individuellen Totemismus. Zunächst bleiben diese Tatsachen aus einem bestimmten Blickwinkel weiterhin soziale Phänomene und bilden Institutionen: In bestimmten Stämmen gehört es zum festen Glauben, dass jedes Individuum sein eigenes Totem besitzt; ähnlich besitzt in Rom jeder Bürger seinen genius, besitzt im Katholizismus jeder Gläubige einen Heiligen als Namenspatron. Doch da ist noch mehr: Diese Phänomene rühren schlicht daher, dass eine sozialfundierte Institution in den Bewusstseinen der Einzelnen eine Brechung und Verzerrung erfahren hat. Wenn jeder Krieger neben seinem Clantotem ein individuelles Totem besitzt, wenn der eine sich den Leoparden verwandt glaubt, der andere sich mit den Raben verbunden fühlt, dann deshalb, weil jeder Einzelne sich sein eigenes Totem nach dem Bild des Clantotems gebildet hat.

37 Erkennbar ist nun, was wir unter kollektiven Vorstellungen verstehen und inwieweit wir sagen können, dass die sozialen Phänomene Bewusstseinsphänomene sein können, ohne deshalb Phänomene des individuellen Bewusstseins zu sein. Wir konnten auch sehen, welche Art Beziehungen zwischen den sozialen Phänomenen bestehen. - Wir sind jetzt in der Lage, unsere weiter vorn getroffene Formulierung zur soziologischen Erklärung zu präzisieren, wonach sie darin besteht, von einem sozialen Phänomen zum anderen sozialen Phänomen voranzuschreiten. Aus dem bisher Gesagten ist zu ersehen, dass es zwei große Ordnungen sozialer Phänomene gibt: die Tatsachen der sozialen Struktur, das heißt die Formen der Gruppe, die Art und Weise, wie die Elemente darin angeordnet sind; und die kollektiven Vorstellungen, in denen die Institutionen gegeben sind. Darauf gegründet kann man sagen, dass jede soziologische Erklärung unter eine 
der folgenden drei Rahmenbedingungen fällt: entweder 1. verbindet sie eine kollektive Vorstellung mit einer anderen kollektiven Vorstellung, beispielsweise das Strafmaß mit privater Rache; oder 2. sie verknüpft eine kollektive Vorstellung mit einem Tatbestand der sozialen Struktur als deren Ursache; so wird die Bildung von Städten als Ursache für die Entwicklung eines städtischen Rechts angesehen, dem Ursprung eines Großteils unseres Eigentumssystems; oder aber 3. sie verknüpft Tatsachen der sozialen Struktur mit den sie determinierenden kollektiven Vorstellungen: so haben bestimmte mythische Vorstellungen die Migrationsbewegungen der Hebräer, der islamischen Araber beherrscht; die von den großen Städten ausgeübte Faszination ist eine Ursache für die Landflucht. - Tatsächlich kann der Eindruck entstehen, als drehten sich derartige Erklärungen im Kreis, insofern die Gruppenformen bald als Wirkungen, bald als Ursachen der kollektiven Vorstellungen dargestellt werden. Doch dieser durchaus reale Zirkel impliziert keineswegs eine Petitio Principii: er ist den Dingen selbst inhärent. Nichts ist vergeblicher als sich zu fragen, ob Ideen die Gesellschaft hervorgerufen oder ob Gesellschaften, einmal entstanden, die kollektiven Ideen hervorgebracht haben: es sind voneinander nicht zu trennende Phänomene, zwischen denen einen logischen oder chronologischen Vorrang herzustellen es keinen Grund gibt.

Die so verstandene soziologische Erklärung verdient es also keineswegs, dass man ihr, wie es zuweilen geschieht, Materialismus vorwirft. Zunächst einmal ist sie frei von aller Metaphysik, sei sie materialistisch oder andersgeartet. Zudem weist sie der psychischen Komponente des sozialen Lebens, den kollektiven Glaubensüberzeugungen und Gefühlen, eine ausschlaggebende Rolle zu. Andererseits entzieht sie sich den Mängeln der Ideologie. Die kollektiven Vorstellungen dürfen nicht so aufgefasst werden, als entwickelten sie sich aus sich selbst heraus, kraft einer Art inneren Dialektik, die sie zwingen würde, sich immer weiter zu entschlacken, sich einem Vernunftideal zu nähern. Familie, Strafrecht haben sich nicht infolge von Vernunftfortschritten eines Denkens verändert, das nach und nach seine ursprünglichen Irrtümer korrigieren würde. Kollektive Meinungen, Gefühle ändern sich nur, wenn die sozialen Zustände, von denen sie abhängen, sich ebenfalls verändern. Eine beliebige soziale Veränderung, etwa der Übergang vom Polytheismus zum Monotheismus, ist nicht mit dem Hinweis erklärt, dass sie einen Fortschritt darstellt, wahrer oder moralischer ist, denn die Frage ist doch, was genau verursacht hat, dass die Religion in diesem Sinne wahrer oder moralischer geworden ist, das heißt in Wirklichkeit, was genau sie dazu gebracht hat, das zu werden, was sie ist. Die sozialen Phänomene werden ebenso wenig wie die Phänomene der Natur aus sich selbst heraus angetrieben. Die Ursache einer sozialen Tatsache muss immer außerhalb ihrer gesucht werden. Mit anderen Worten, Aufgabe des Soziologen ist nicht, irgendein Fortschritts-, allgemeines Entwicklungsgesetz zu finden, das die Vergangenheit beherrschte und die Zukunft vorbestimmte. Es gibt kein einzelnes, universelles Gesetz der sozialen Phänomene. Es gibt eine Vielzahl von Gesetzen ungleicher Allgemeinheit. In der Soziologie wie in jeder Wissenschaft heißt erklären also, mehr oder weniger bruchstückhafte Gesetze zu entdecken, das heißt bestimmte Tatsachen entsprechend bestimmten Beziehungen miteinander zu verknüpfen. 


\section{Methode der Soziologie}

39 Abhandlungen über die soziologische Methode gibt es in großer Zahl. Im Allgemeinen sind sie mit allen möglichen philosophischen Betrachtungen zur Gesellschaft, zum Staat usw. vermischt. $\mathrm{Zu}$ den ersten Werken, in denen die Methode der Soziologie eingehend untersucht wurde, gehören die von Comte und Stuart Mill. Doch ungeachtet ihrer Bedeutung verblieben die methodologischen Beobachtungen dieser beiden Philosophen, wie die entsprechende Wissenschaft, die sie begründen wollten, auf der Stufe höchster Allgemeinheit. Vor kurzem hat Durkheim genauer zu bestimmen versucht, wie die Soziologie bei der Untersuchung der besonderen Tatsachen vorzugehen hat.

40 Sicher kann es nicht darum gehen, die Regeln der soziologischen Methode umfassend und endgültig zu formulieren. Denn eine Methode unterscheidet sich nur in abstrakter Hinsicht von der Wissenschaft selbst. Sie gliedert und organisiert sich allein nach Maßgabe der Fortschritte dieser Wissenschaft. Unsere Absicht hier ist lediglich, einige bereits durch Gebrauch eingebürgerte wissenschaftliche Verfahren zu analysieren.

\section{Definition}

41 Wie jede Wissenschaft muss die Soziologie die Untersuchung eines Problems mit einer Definition beginnen. Zunächst ist der Forschungsbereich anzugeben und zu begrenzen, um zu wissen, wovon die Rede ist. Diese Definitionen erfolgen vorab und sind infolgedessen provisorisch. Weder können noch sollen sie die Essenz der zu untersuchenden Phänomene ausdrücken, sondern sie nur klar und deutlich bezeichnen. Bei aller Äußerlichkeit sind sie doch unverzichtbar. Mangels Definitionen setzt sich jede Wissenschaft Konfusionen und Fehlern aus. Ohne sie würde ein Soziologe im Untersuchungsverlauf ein und demselben Wort unterschiedliche Bedeutungen zuschreiben. Er würde damit gravierende Fehler begehen: So benutzen viele Autoren im Rahmen der Familientheorie unterschiedslos die Begriffe Stamm, Dorf, Clan zur Bezeichnung ein und derselben Sache. Zudem können sich Wissenschaftler ohne Definitionen in Diskussionen untereinander nicht verständigen, da sie nicht über dasselbe sprechen. Ein Gutteil der Debatten, die die Familien- und Heiratstheorie ausgelöst hat, geht auf das Fehlen von Definitionen zurück: So nennen die einen Monogamie, was die anderen nicht mit diesem Wort bezeichnen, vermischen die einen das Rechtswesen, das die Monogamie fordert, mit der schlichten faktischen Monogamie; andere wiederum unterscheiden diese in Wirklichkeit sehr unterschiedlichen Ordnungen von Tatsachen.

Natürlich sind derartige Definitionen konstruiert. In ihnen wird eine Reihe von Tatsachen zusammengefasst und bezeichnet, deren grundlegende Ähnlichkeit man vorhersieht. Dennoch sind es keine apriorischen Konstruktionen, vielmehr das Ergebnis eines ersten Arbeitsvorgangs, einer ersten schnellen Übersicht über die Fakten, deren gemeinsame Qualitäten ausgemacht werden. Ihr vorrangiger Zweck ist es, die Begriffe des Alltagsverstandes durch einen ersten wissenschaftlichen Begriff zu ersetzen. Denn tatsächlich gilt es in erster Linie, sich von den gängigen Vorurteilen zu lösen, die in der Soziologie gefährlicher sind als in jeder anderen Wissenschaft. Ohne Überprüfung darf keine übliche Klassifizierung als wissenschaftliche Definition postuliert werden. Viele noch in manchen Sozialwissenschaften gebräuchlichen Ideen 
scheinen weder rational noch faktisch begründet zu sein und sollten aus einer vernunftbasierten Terminologie verbannt werden. Zum Beispiel entspricht der Begriff des Heidentums und selbst der des Fetischismus nichts Realem. In anderen Fällen führt eine ernsthafte Forschung dazu, das zu vereinen, was der Volksmund unterscheidet, oder das zu unterscheiden, was nämlicher Volksmund vereint. Zum Beispiel hat die Religionswissenschaft die Tabus der Unreinheit und der Reinheit einer Art zugeschlagen, weil sie Tabus sind; umgekehrt hat sie sorgfältig die Beerdigungsriten und den Ahnenkult unterschieden.

Diese Definitionen sind umso genauer und sachhaltiger, je mehr man sich bemüht, die objektiven Merkmale der Dinge zu erfassen. Als objektive Merkmale werden jene bezeichnet, die diesem oder jenem sozialen Phänomen an sich selbst eignen, das heißt jene, die nicht von unseren Gefühlen und persönlichen Meinungen abhängen. So dürfen wir die Opferung nicht nach unserer mehr oder weniger durchdachten Idee dieses Ritus definieren, sondern anhand der äußeren Merkmale, die sie als soziale und religiöse Tatsache, äußerlich und unabhängig von uns, zeigt. In dieser Weise begriffen, wird die Definition zu einem wichtigen Moment der Forschung. Diese Merkmale, anhand deren das zu untersuchende soziale Phänomen definiert wird, sind zwar äußerlich, aber entsprechen damit nicht weniger den wesentlichen Merkmalen, die die Analyse offenbart. Zutreffende Definitionen können somit den Weg zu bedeutsamen Entdeckungen eröffnen. Wird Verbrechen als Akt definiert, der einen Anschlag auf die Rechte der Individuen darstellt, dann sind die einzigen als solche bezeichneten Handlungen heutzutage Tötungsdelikte, Diebstahl usw. Wird es dagegen als eine Handlung definiert, die eine organisierte Reaktion des Kollektivs herausfordert, versteht man laut Definition darunter zwangsläufig alle wirklich ursprünglichen Formen des Verbrechens, insbesondere die Verletzung der religiösen Regeln, des Tabus zum Beispiel.

Schließlich stellen diese Vorabdefinitionen eine erstrangige wissenschaftliche Gewähr dar. Sind sie erst einmal postuliert, verpflichten und binden sie den Soziologen. Sie erhellen sein gesamtes Vorgehen, ermöglichen wirksame Kritik und Diskussion. Dank ihrer drängt sich der Untersuchung eine ganze Reihe wohlbestimmter Tatsachen auf, und die Erklärung muss sie alle berücksichtigen. Umgangen werden damit all jene kapriziösen Argumentationen, in denen ein Autor nach Belieben von einem Thema aufs andere abschweift und sich seine Belege aus den heterogensten Tatsachenkategorien holt. Zudem vermeidet man einen Fehler, den noch die besten soziologischen Arbeiten begehen, zum Beispiel Frazer hinsichtlich des Totemismus. Dieser Fehler besteht darin, nur die Tatsachen zusammenzufassen, die die These bestätigen, und nicht weiter nach den ihr widersprechenden zu forschen. Im Allgemeinen lässt man es daran vermissen, alle Fakten in die Theorie zu integrieren, und erfasst nur diejenigen, die sich exakt decken. Mit guten Anfangsdefinitionen nun bieten sich zwangsläufig alle sozialen Tatsachen gleicher Ordnung dem Beobachter dar, und er ist gehalten, nicht nur über die Übereinstimmungen, sondern auch über die Abweichungen Aufschluss zu geben.

\section{Beobachtung der Tatsachen}

45 Wie gesehen, setzt die Definition eine erste allgemeine Übersicht über die Tatsachen, eine Art provisorische Beobachtung voraus. Jetzt müssen wir über die methodische Beobachtung sprechen, das heißt jene, die jede der erwähnten Tatsachen festlegt. Die Beobachtung der sozialen Phänomene ist, anders als man es auf den ersten Blick 
erwarten würde, kein rein erzählendes Verfahren. Die Soziologie muss mehr tun als nur die Tatsachen beschreiben, sie muss sie in Wirklichkeit erst konstituieren. Zunächst einmal gilt, dass es in der Soziologie wie in jeder anderen Wissenschaft keine rohen Fakten gibt, die man einfach sozusagen abfotografieren könnte. Jede wissenschaftliche Beobachtung bezieht sich auf methodisch ausgewählte und von den anderen abgesonderte, also abstrakte Phänomene. Mehr noch als bei anderen können bei sozialen Phänomenen nicht alle Einzelheiten und alle ihre Beziehungen auf einmal untersucht werden. Sie sind zu komplex, um anders als durch Abstraktion und schrittweise Abspaltung der Schwierigkeiten vorzugehen. Doch auch wenn die soziologische Beobachtung die Tatsachen abstrakt fasst, verfährt sie dabei nicht weniger sorgfältig und ist bemüht, sie genau festzustellen. Nun sind soziale Tatsachen schwer zu erreichen und aus den Dokumenten zu gewinnen. Noch schwieriger ist es, sie zu analysieren und in einigen Fällen ihre annähernden Ausmaße anzugeben. Es bedarf daher besonderer und strenger Beobachtungsverfahren; es bedarf, um im gewohnten Sprachgebrauch zu verbleiben, kritischer Methoden. Selbstverständlich verändert sich der Gebrauch dieser Methoden je nach den variablen Tatsachen, die soziologisch beobachtet werden. So unterscheiden sich die Mittel zur Analyse eines religiösen Ritus und zur Beschreibung der Entwicklung einer Stadt. Doch die geistige Arbeitseinstellung, die Arbeitsmethode bleiben gleich, und so lassen sich die kritischen Methoden nur nach der Beschaffenheit der Dokumente, auf die sie angewendet werden sollen, klassifizieren: die einen sind statistische Dokumente, die fast alle modernen, jüngeren Datums sind; die anderen sind historische Dokumente. Die mit der Verwendung dieser Dokumente aufgeworfenen zahlreichen Probleme sind recht unterschiedlich, und zugleich doch auch analoger Art.

In jeder auf statistisches Material gestützten Arbeit ist es wichtig, ja unverzichtbar, sorgfältig darzustellen, wie man zu den verwendeten Daten gekommen ist. Denn beim gegenwärtigen Zustand der diversen Statistiken - rechtlicher, wirtschaftlicher, demografischer Art usw. - erfordert jedes Dokument einen scharfen kritischen Blick. Schauen wir uns nur einmal die offiziellen Dokumente an, die in der Regel die beste Gewähr bieten. Diese Dokumente müssen in allen ihren Einzelheiten geprüft werden, und die ihrer Sammlung zugrunde liegenden Grundsätze müssen gut bekannt sein. Fehlt es an derartigen Vorkehrungen, besteht das Risiko, an falsche Daten zu gelangen. So ist es unmöglich, die statistischen Angaben über Selbstmord in England zu verwenden, denn in diesem Land werden die meisten Selbstmorde, um der Härte des Gesetzes zu entgehen, als Tod aufgrund von Wahnsinn deklariert; die Statistik ist damit bereits von ihrer Grundlage her korrumpiert. Zudem ist darauf $\mathrm{zu}$ achten, die verfügbaren Daten, die aus unterschiedlichen Quellen stammen, zu vergleichbaren Tatsachen zusammenzuziehen. Da sie nicht in dieser Weise vorgegangen sind, enthalten zum Beispiel viele Arbeiten zur Moralsoziologie gravierende Fehler. Dabei wurden Werte verglichen, die in den verschiedenen europäischen Statistiken nicht dieselbe Bedeutung haben. Denn den Statistiken liegen Kodierungen zugrunde, und die verschiedenen Kodierungen weisen weder dieselbe Klassifikation noch dieselbe Nomenklatur auf; so unterscheidet das englische Recht nicht zwischen fahrlässiger und vorsätzlicher Tötung. Außerdem muss die statistische Beobachtung wie jede wissenschaftliche Beobachtung darauf bedacht sein, so genau und detailliert wie möglich zu sein. Tatsächlich wandelt sich häufig der Charakter eines Tatbestands, wenn eine allgemeine Beobachtung durch eine immer genauere Analyse ersetzt wird. 
So lässt eine Karte der Selbstmorde in den Arrondissements Frankreichs andere Phänomene erkennen als eine je nach Departements.

Hinsichtlich historischer oder ethnografischer Dokumente muss die Soziologie im Großen und Ganzen auf die Verfahren der »historischen Kritik« zurückgreifen. Sie darf sich nicht erfundener Tatsachen bedienen und muss folglich die Richtigkeit der Informationen, auf die sie sich stützt, feststellen. Derartige Verfahren der Kritik sind umso notwendiger, als den Soziologen häufig nicht ohne Grund vorgeworfen worden ist, sie zu vernachlässigen. So wurden zum Beispiel ohne ausreichendes Urteilsvermögen Berichte und Angaben von Reisenden und Ethnografen benutzt. Kenntnis der Quellen und strenge kritische Prüfung hätten es den Soziologen erlaubt, ihre Theorien zu den elementaren Formen des sozialen Lebens auf eine unbestreitbare Grundlage $\mathrm{zu}$ stellen. Im Übrigen ist $\mathrm{zu}$ hoffen, dass die Fortschritte der Geschichtswissenschaft und der Ethnografie durch Beschaffung einwandfreier Informationen die Arbeit erleichtern werden. Die Soziologie darf sich von den Fortschritten der beiden Fächer alles erhoffen. Doch auch wenn der Soziologe dieselben kritischen Ansprüche hat wie der Historiker, muss er, da er die Tatbestände aus einer anderen geistigen Einstellung und im Hinblick auf ein anderes Ziel untersucht, seine Kritik nach davon unterschiedenen Grundsätzen führen. Zunächst einmal untersucht er, soweit es geht, nur soziale, tiefgehende Tatsachen; und dass entsprechende Anliegen sich in jüngster Zeit auch in den Geschichtswissenschaften bemerkbar machen, ist hinlänglich bekannt; denn dort fehlt es zum Beispiel an ausreichenden und treffenden historischen Studien zur Wirtschaftsorganisation selbst unseres eigenen Landes. Sodann stellt die Soziologie an die Tatsachen keine unlösbaren Fragen, deren Beantwortung zudem auch nur geringe Erklärungskraft hat. So ist es angesichts des Fehlens gesicherter Monumente nicht unbedingt nötig, den Rigveda präzise zu datieren: es ist unmöglich und im Grunde auch gleichgültig. Um sich einer sozialen Tatsache, etwa eines Betrituals, in der Soziologie zu bedienen, muss diese nicht zeitlich verortet sein; Hauptsache ist, dass man die ihr vorausgehenden, zeitgleich mit ihr bestehenden und die ihr folgenden Tatsachen, mit anderen Worten, den sie umgebenden sozialen Rahmen kennt. Schließlich sucht der Soziologe nicht ausschließlich nach dem, was an einer jeden Tatsache einzigartig ist. Nachdem sie sich bisher vor allem der Biografie der großen Männer und Tyrannen gewidmet haben, versuchen sich die Historiker jetzt vorrangig an der kollektiven Biografie. Sie widmen sich den besonderen Ausprägungen der Sitten, der Glaubensartikel der kleinen wie großen Gruppen. Sie forschen nach dem Trennenden, dem einzigartig Machenden und suchen gewissermaßen das in jeder Zivilisation nachzuzeichnen, was an Unaussprechlichem vorliegt. So glaubt man beispielweise in der Regel, dass das Studium der vedischen Religion ausschließlich Kennern des Sanskrits vorbehalten sei. Dem Soziologen geht es im Gegenteil darum, das Allgemeine wie das Charakteristische in den sozialen Tatsachen ausfindig zu machen. In seinen Augen soll eine sachgemäß durchgeführte Beobachtung ein genau bestimmtes Residuum ergeben, einen hinreichend adäquaten Ausdruck des beobachteten Faktums. Um sich einer bestimmten sozialen Tatsache $\mathrm{zu}$ bedienen, ist die umfassende Kenntnis einer Geschichte, einer Sprache oder einer Zivilisation nicht notwendig. Die relative, aber genaue Kenntnis dieser Tatsache genügt, damit sie in das System, das die Soziologie erbauen will, Eingang finden kann und finden soll. Und wenn es in vielen Fällen unerlässlich ist, dass der Soziologe bis zu den letzten Quellen gehen muss, dann sind daran nicht die Tatsachen schuld, sondern die Historiker, die keine sachgemäße, 
wirkliche Analyse derselben anzufertigen wussten. Die Soziologie verlangt sichere, unpersönliche und für jeden, der die gleichen Tatbestände untersucht, verwendbaren Beobachtungen. Die Tatsachen sind in ihren Einzelheiten und ihren Begleitumständen unendlich, von niemanden je zu erschöpfen; die reine Geschichtswissenschaft wird nie aufhören $\mathrm{zu}$ beschreiben, $\mathrm{zu}$ nuancieren, $\mathrm{zu}$ kontextualisieren. Eine sorgfältig durchgeführte Beobachtung, eine sachgemäß untersuchte und in ihrer Gänze analysierte Tatsache dagegen verlieren jeden zeitlichen Index, genauso wie eine medizinische Beobachtung oder ein außergewöhnlicher Laborversuch. Eine wissenschaftlich beschriebene soziale Tatsache wird zu einem wissenschaftlichen Element und hört auf, diesem oder jenem Land, dieser oder jener Epoche anzugehören. Sie ist, kraft der wissenschaftlichen Beobachtung, gewissermaßen außerhalb von Zeit und Raum gestellt.

\section{Systematisierung der Tatsachen}

Wie jede andere Wissenschaft, so spekuliert auch die Soziologie nicht über reine Ideen und begnügt sich mit der Registrierung der Tatsachen. Sie versucht, diese in ein rationales System einzuordnen. Sie sucht deren Beziehungen herauszufinden und sie so intelligibel zu machen. Wir müssen jetzt noch erklären, mittels welcher Verfahren diese Beziehungen bestimmt werden können. Manchmal, übrigens recht selten, findet man sie gewissermaßen schon fix und fertig vor. Tatsächlich gibt es in der Soziologie wie in anderen Wissenschaften derart typische Tatsachen, dass sich anhand einer sachgerechten Analyse sofort unverhoffte Beziehungen entdecken lassen. Auf eine derartige Tatsache sind Fison und Howitt gestoßen, als sie bei der Erklärung des Systems der Verwandtschaft und der exogamen Klassen in bestimmten australischen Stämmen ein neues Licht auf die primitiven Familienformen geworfen haben. Doch im Allgemeinen stoßen wir nicht direkt, durch schlichte Beobachtung, auf derartige zentrale Tatsachen. Es muss also eine ganze Reihe spezieller methodischer Verfahren aufgeboten werden, um die bestehenden Beziehungen zwischen den Fakten festzustellen. Hier befindet sich die Soziologie gegenüber den anderen Wissenschaften in einer unterlegenen Situation. Das Experiment ist ihr verwehrt; man kann typische soziale Tatsachen nicht absichtlich hervorrufen und dann untersuchen. Man muss also auf den Vergleich verschiedener, aber kategorial gleicher sozialer Tatsachen aus verschiedenen Gesellschaften zurückgreifen und so deren Wesenskern herauszuschälen suchen. Im Grunde kann ein sachgemäß durchgeführter Vergleich in der Soziologie zu äquivalenten Ergebnissen wie beim Experiment führen. Man geht in etwa wie ein Zoologe vor, wie Darwin vorgegangen ist. Mit einer Ausnahme konnte dieser keine richtigen Experimente durchführen und abgewandelte Arten erzeugen; er musste ein allgemeines Schaubild dessen erstellen, was er über deren Ursprung wusste; und durch den methodischen Vergleich dieser Fakten konnte er dann seine Hypothesen entwickeln. Ähnlich konnte Morgan in der Soziologie die Hypothese des Clans mit mütterlicher Abstammung aufstellen, nachdem er die Identität des Familiensystems der Irokesen, Hawaiianer, Fidschianer usw. ausgemacht hatte. Im Übrigen hat der Vergleich der sozialen Tatsachen, wenn er von wirklichen Wissenschaftlern durchgeführt wurde, stets gute Ergebnisse erbracht. Selbst wenn, wie in den Arbeiten der englischen anthropologischen Schule, theoretisch nichts davon geblieben ist, hat er zumindest zu einer allgemeinen Klassifizierung eines Großteils von Tatsachen geführt. 
Zudem ist man bemüht, sollte es jedenfalls sein, den Vergleich immer exakter zu gestalten. Einige Autoren, darunter Tylor und Steinmetz, haben sogar, der eine in Bezug auf Heirat, der andere im Hinblick auf Strafe und Endokannibalismus, eine statistische Methode vorgeschlagen und angewandt. Hier äußern sich die Übereinstimmungen und Unterschiede zwischen den festgestellten Fakten in Zahlen. Allerdings sind die dabei erzielten Resultate alles andere als befriedigend, denn die dabei herangezogenen Tatsachen stammen aus den unterschiedlichsten und heterogensten Gesellschaften und sind in Dokumenten höchst ungleicher Güte festgehalten. Auf diese Weise wird der Anzahl der Befunde und der gesammelten Fakten eine übermäßige Bedeutung beigemessen, dagegen auf die Qualität dieser Befunde, ihre Zuverlässigkeit, den demonstrativen Wert und die Vergleichbarkeit der Fakten weniger geachtet. Wahrscheinlich ist es vorteilhafter, auf den Anspruch der Genauigkeit zu verzichten und sich an elementare, aber strenge Vergleiche zu halten. Zum ersten ist es wichtig, Tatsachen derselben Ordnung zu vergleichen, das heißt jene, die unter die am Anfang der Arbeit postulierte Definition fallen. So ist man gut beraten, in einer Theorie der Familie, in Bezug auf den Clan, nur auf diesen bezogene Tatsachen zusammenzufassen und mit ihnen keine ethnografischen Auskünfte zu vermischen, die in Wirklichkeit den Stamm oder die lokale Gruppe betreffen, welche allerdings oft mit dem Clan verwechselt werden. Zum zweiten müssen die verglichenen Tatsachen zu sorgsam gebildeten Reihen geordnet werden. Anders gesagt, man ordnet die verschiedenen Formen, in denen sie sich zeigen, in einer ganz bestimmten Weise an, entweder nach zunehmender oder abnehmender Komplexität oder gemäß irgendeiner anderen Variation. Zum Beispiel wird man in einer Theorie der patriarchalen Familie die hebräische Familie unterhalb der griechischen und diese unterhalb der römischen Familie einordnen. Zum dritten wird man im Blick auf diese Reihe weitere, auf gleiche Weise konstruierte, aber aus anderen sozialen Tatsachen bestehende Reihen aufstellen. Und aus den erkennbaren Beziehungen zwischen diesen Reihen lassen sich die Hypothesen gewinnen. Beispielsweise lässt sich die Entwicklung der patriarchalen Familie mit der des städtischen Gemeinwesens verknüpfen - sichtbar wird dabei etwa, wie von den Hebräern zu den Griechen und von diesen zu den Römern mit der wachsenden Dichte des Gemeinwesens die väterliche Macht zunimmt.

\section{Der wissenschaftliche Charakter der soziologischen Hypothesen}

50 So gelingt es, mit Hilfe angemessen beobachteter Tatsachen und in Bezug auf klar definierte Probleme Hypothesen zu entwickeln und zu verifizieren. Natürlich sind diese Hypothesen nicht zwangsläufig richtig; viele der uns heute evident erscheinenden werden eines Tages fallengelassen. Doch auch wenn nicht allen der Charakter absoluter Wahrheit eignet, weisen sie doch die Merkmale der wissenschaftlichen Hypothese auf. Erstens sind sie alle wirklich erklärend; sie benennen das Warum und das Wie der Dinge. Eine rechtliche Regel wie die Haftpflicht wird hier nicht mit dem klassischen "Willen des Gesetzgebers" erklärt oder den allgemeinen "Tugenden« der Menschennatur, die angeblich diese Institution vernünftigerweise geschaffen hat. Erklärt wird sie vielmehr anhand der gesamten Entwicklung des Systems der Haftung. Zweitens weisen sie durchaus den der methodischen Induktion zugehörigen Charakter der Notwendigkeit und dementsprechend der Allgemeinheit auf, der in einigen Fällen vielleicht sogar die Prognose erlaubt. Beispielweise kann man fast als Gesetz postulieren, dass die rituellen Praktiken im Laufe der Entwicklung der 
universalistischen Religionen tendenziell seltener und spiritueller werden. Drittens, und das ist unseres Erachtens der wichtigste Punkt, sind diese Hypothesen in höchstem Maße kritisierbar und verifizierbar. In einer wirklichen soziologischen Arbeit kann jeder behandelte Punkt der Kritik unterzogen werden. Man ist fern jenes Feinstaubs an Fakten oder jenen Phantastereien aus Ideen und Wörtern, die der Öffentlichkeit häufig als Soziologie gilt, wo aber weder präzise Gedanken noch ein rationales System, noch eine dichte Studie der Tatsachen zu finden sind. Die Hypothese wird zu einem Element der präzisen Diskussion; die Methode, die Anfangsdefinition, die angeführten Fakten, die vorgenommen Vergleiche lassen sich bestreiten, korrigieren; so, dass in der Wissenschaft Fortschritte möglich sind.

51 Hier ist einem Einwand vorzugreifen. Man könnte versucht sein zu sagen, dass die Soziologie vor ihrem Aufbau eine umfassende Bestandsaufnahme aller sozialen Tatsachen erstellen müsse. Dem Theoretiker der Familie wäre dementsprechend abzuverlangen, alle diese Frage betreffenden ethnografischen, historischen, statistischen Dokumente vollständig zu sondieren. Tendenzen dieser Art sind in unserer Wissenschaft durchaus zu befürchten. Scheu vor den Fakten ist genauso gefährlich wie übertriebener Wagemut, der kleinmütige Verzicht des Empirismus ist genauso verhängnisvoll wie übereilte Generalisierungen. Zum ersten, die Wissenschaft fordert zwar immer vollständigere Faktenübersichten, verlangt aber nirgends eine totale Bestandsaufnahme, die im Übrigen unmöglich ist. Um eine Theorie der Verdauung zu entwickeln, hat der Biologe nicht gewartet, bis er alle entsprechenden Fakten auf allen Stufen des Tierreichs beobachtet hat. Der Soziologe muss genauso vorgehen; auch er muss nicht alle sozialen Tatsachen einer bestimmten Kategorie von Grund auf kennen, um eine entsprechende Theorie zu erarbeiten. Er muss sich unverzüglich an die Arbeit machen. Provisorischen, aber sorgsam aufgelisteten und präzise gefassten Kenntnissen entsprechen provisorische Hypothesen. Vorgenommene Verallgemeinerungen, vorgeschlagene Systeme gelten für den Augenblick für alle bekannten und unbekannten Tatsachen gleicher Ordnung so gut wie explizierte Tatsachen. Man muss nur bereit sein, die Theorien in dem Maße zu ändern, wie neu hinzugekommene Fakten bekannt werden oder die von Tag zu Tag genauere Wissenschaft neue Aspekte an den bekannten Fakten entdeckt. Jenseits einer solchen immer stärkeren Annäherung an die Phänomene gibt es nur dialektische Diskussionen und gelehrte Enzyklopädien, die einen wie die anderen ohne wirklichen Nutzen, da sie keine Erklärung anbieten. Und wenn die Induktion methodisch vorgenommen wurde, können die vom Soziologen gewonnenen Resultate gar nicht ohne Realitätskern sein. Die Hypothesen bringen Tatsachen zum Ausdruck, und folglich eignet ihnen immer zumindest ein Stück Wahrheit: Die Wissenschaft kann sie vervollständigen, korrigieren, verändern, wird es aber nie daran fehlen lassen, sie zu benutzen.

\section{Gliederungen der Soziologie}

Die Soziologie erhebt den Anspruch, Wissenschaft und mit der etablierten wissenschaftlichen Tradition verbunden zu sein. Sie ist deshalb aber nicht minder unabhängig von den existierenden Klassifikationen. Sie kann die Arbeit anders aufteilen, als es bisher geschehen ist.

Erstens betrachtet die Soziologie eine gewisse Anzahl von Problemen als die ihren, die bis dato in das Ressort der Wissenschaften gehörten, die keine »Sozialwissenschaften« 
sind. Sie demontiert diese Wissenschaften, überlässt ihnen ihren ureigenen Gegenstand und behält für sich alle die Tatsachen, die ausschließlich sozialer Ordnung sind. So behandelte etwa die Geografie bislang die Fragen der Grenzen, der Kommunikationswege, der sozialen Dichte usw. Doch das sind keine Fragen der Geografie, vielmehr der Soziologie, denn es handelt sich nicht um kosmische Phänomene, vielmehr um solche, die mit der Natur der Gesellschaften in Zusammenhang stehen. Analog eignet sich die Soziologie bereits bestehende Resultate der Kriminalanthropologie $\mathrm{zu}$ einigen Phänomenen an, die keine somatischen Phänomene sind, sondern soziale Tatsachen.

Zweitens, unter den gemeinhin als "Sozialwissenschaften« bezeichneten Disziplinen gibt es einige, die nicht im buchstäblichen Sinn Wissenschaften sind. Ihre Einheit ist künstlich, und es ist Aufgabe der Soziologie, sie aufzulösen. Dazu gehören Statistik und Ethnografie, die beide als gesonderte Wissenschaften betrachtet werden, während sie lediglich entsprechend ihren jeweiligen Verfahren die verschiedensten Phänomene untersuchen und in Wirklichkeit in die Zuständigkeit verschiedener Teile der Soziologie fallen. Wie gesehen, stellt die Statistik nur eine Methode zur Beobachtung verschiedenster Phänomene des modernen sozialen Lebens dar. Demografische, moralische, ökonomische Phänomene: sie alle werden heute unterschiedslos von der Statistik untersucht. Uns zufolge sollte es hier keine Statistiker geben, sondern Soziologen, die zur Untersuchung der moralischen, ökonomischen usw. Phänomene, zur Untersuchung der Gruppen Statistik der Moral, der Ökonomie, der Demografie betreiben. Gleiches gilt für die Ethnografie. Deren einzige Daseinsberechtigung ist es, sich dem Studium der Phänomene zu widmen, die in den sogenannten "wilden" Nationen vorkommen. Auch sie untersucht unterschiedslos Phänomene der Moral, des Rechts, der Religion, der Techniken, der Künste usw. Die Soziologie dagegen unterscheidet nicht von Natur aus zwischen den Institutionen der "wilden" Völkerschaften und jenen der »barbarischen« oder »zivilisierten« Nationen. Sie nimmt in ihre Definitionen die elementarsten wie die am stärksten entwickelten Tatsachen auf. In einer Untersuchung der Familie oder der Strafe zum Beispiel wird sie sich auferlegen, ebenso die »ethnografischen« wie die »historischen« Fakten in Betracht zu ziehen, die gleichermaßen soziale Tatsachen sind und sich lediglich danach unterscheiden, wie sie jeweils beobachtet werden.

Die Soziologie dagegen übernimmt die großen Gliederungen und macht sie zu ihren eigenen, die bereits von den verschiedenen vergleichenden Wissenschaften der Institutionen, deren Erbin sie zu sein beansprucht, wahrgenommen worden waren: Wissenschaften vom Recht, von den Religionen, Politische Ökonomie usw. Unter diesem Gesichtspunkt lässt sie sich leicht in spezielle Soziologien aufgliedern. Aber bei dieser Übernahme der Aufteilung folgt sie nicht sklavisch den üblichen Klassifikationen, die mehrheitlich empirischen oder praktischen Ursprungs sind, wie etwa in der Rechtswissenschaft. Vor allem errichtet sie zwischen den Tatsachen nicht jene undurchdringlichen Trennwände, die gewöhnlich zwischen den speziellen Wissenschaften herrschen. Der Soziologe, der rechtliche und moralische Tatbestände untersucht, muss zu ihrem Verständnis häufig auf religiöse Phänomene zurückgehen. Wer das Eigentum untersucht, muss dieses Phänomen unter seinem zweifachen Aspekt, dem rechtlichen und dem ökonomischen, betrachten, während diese beiden Seiten ein und desselben Tatbestands gewöhnlich von Wissenschaftlern unterschiedlicher Provenienz untersucht werden. 
Während die Soziologie sich also eng an die Wissenschaften anlehnt, die ihr vorausgegangen sind, sich deren Ergebnisse zu eigen macht, verändert sie gleichzeitig deren Klassifikationen. $\mathrm{Zu}$ bemerken war übrigens in den letzten Jahren, dass alle Sozialwissenschaften die Tendenz aufweisen, sich fortschreitend der Soziologie anzuschließen; mehr und mehr werden sie zu speziellen Teilen ein und derselben Wissenschaft. Doch da diese sich zu einer wirklichen Wissenschaft konstituiert, mit einer bewussten Methode, verändert sie die Forschung bis in ihren Geist hinein und kann damit zu neuen Ergebnissen führen. Wenn daher auch viele Resultate bewahrt werden können, kann doch nicht jedes Teilgebiet der Soziologie mit den verschiedenen bestehenden Sozialwissenschaften zur Deckung kommen. Diese verändern sich aus sich selbst heraus, und die Einführung der soziologischen Methode hat in der Art und Weise, die sozialen Phänomene zu untersuchen, bereits einen Wandel vollzogen und wird dies auch weiterhin tun.

Die sozialen Phänomene gliedern sich in zwei große Ordnungen. Zum einen gibt es die Gruppen und ihre Strukturen. Entsprechend gibt es ein Spezialgebiet der Soziologie, in dem Gruppen untersucht werden, die Anzahl der Individuen, aus denen sie bestehen, und deren jeweilige räumliche Verteilung: das ist die Sozialmorphologie. Zum anderen gibt es jene sozialen Tatsachen, die innerhalb der Gruppen $\mathrm{zu}$ finden sind: die Institutionen und die kollektiven Vorstellungen. Diese bilden die eigentlichen großen Funktionen des sozialen Lebens. Jede dieser Funktionen - die religiöse, rechtliche, ökonomische, ästhetische usw. - muss zunächst gesondert untersucht und einer Reihe relativ unabhängiger Forschungen unterzogen werden. Unter diesem Gesichtspunkt gibt es mithin eine Religionssoziologie, eine Moral- und Rechtssoziologie, eine Techniksoziologie usw. Wenn dann alle diese speziellen Untersuchungen vorliegen, wird es möglich, einen letzten Teil der Soziologie zu entwickeln, die allgemeine Soziologie, deren Forschungsgegenstand das wäre, was die Einheit aller sozialen Phänomene ausmacht.

\section{BIBLIOGRAPHIE}

\section{Zur Geschichte der Soziologie}

Bouglé, C. (1896): Les sciences sociales en Allemagne, Paris: Alcan.

Durkheim, É. (1887): »Les sciences morales en Allemagne«, Revue philosophique.

Durkheim, É. (1900): „La sociologie en France au XIX ${ }^{\mathrm{e}}$ siècle«, Revue bleu, Mai.

Espinas, A. V. (1867): Sociétés animales; dt.: Die thierischen Gesellschaften. Eine vergleichens-

psychologische Untersuchung. Nach der vielfach erweiterten zweiten Auflage unter Mitwirkung des Verfassers deutsch herausgegeben von W. Schloesser. Braunschweig: Vieweg und Sohn, 1879.

Fouillée, A. (1885): La science sociale contemporaine, Paris: Hachette.

Groppali, A. (1900): »La sociologie en Amérique«, Annales de l'Institut international de sociologie. 
Lévy-Bruhl, L. (1900): La philosophie d'Auguste Comte, Paris: Alcan.

\section{Zur Soziologie im Allgemeinen}

Comte, A. (1839-1842): Cours de philosophie positive, Paris: Bachelier; dt. Teilübersetzung: Die Soziologie. Positive Philosophie, Stuttgart: Kröner, 1974.

De Greef, G. (1886-1889): Introduction à la sociologie, Paris: Alcan.

De Greef, G. (1895): Le transformisme social: essai sur le progrès et le regrès des sociétés, Paris: Alcan.

Espinas, A. V. (1867): Sociétés animales; dt.: Die thierischen Gesellschaften. Eine vergleichenspsychologische Untersuchung. Nach der vielfach erweiterten zweiten Auflage unter Mitwirkung des Verfassers deutsch herausgegeben von W. Schloesser. Braunschweig: Vieweg und Sohn, 1879.Giddings, F. H. (1970 [1896]): The Principles of Sociology, New York: Johnson; dt.: Prinzipien der Soziologie, Leipzig: Kröner, 1911.

Gumplowicz, L. (1885): Grundriß der Soziologie, Wien: Manz.

Schäffle, A. E. F. (2016 [1896]): Bau und Leben des sozialen Körpers, Nachdruck, Norderstedt: Hansebooks $\mathrm{GmbH}$.

Small, A. W. (1971 [1894]): An Introduction tot he Study of Society, Dubuque: Brown Reprints.

Spencer, H. (1876-1896): The Principles of Sociology, 3 Bde., New York: D. Appleton and Co.; dt.: Die Principien der Sociologie, 4 Bde., Stuttgart: Schweizerbart, 1877-1888.

Spencer, H. (1969 [1873]): The Study of Sociology, Ann Arbor: University of Michigan Press.

Spencer, H. (1970 [1851]): Social Statics, Robert Schalkenbach Foundation.

Tarde, G. (1890-1895): Les lois de l'imitation: étude sociologique, Paris: Alcan; dt.: Die Gesetze der Nachahmung, übers. von Jadja Wolf, Frankfurt am Main: Suhrkamp, 2003.

Tarde, G. (1895): La logique sociale, Paris: G. Baillière \& C.

Tönnies, F. (1887): Gemeinschaft und Gesellschaft. Abhandlung des Communismus und des Socialismus als empirischer Culturformen, Leipzig: Fues.

Ward, L. F. (1883): Dynamic Sociology or Applied Social Science as Based Upon Statical Sociology and the Less Complex Sciences, New York: Appleton.

\section{Die Hauptwerke der organizistischen Schule sind u. a.:}

Demoor, J. / Massart, J. / Vandervelde, É. (1897): L'Évolution régressive en biologie et en sociologie, Paris: Alcan.

Massart, J. / Vandervelde, É. (1893): Parasitisme organique et parasitisme social, Paris: C. Carré. Novicow, J. (1893): Les luttes entre les sociétés humaines, Paris: Alcan.

Novicow, J. (1897): Conscience et volonté sociales, Paris: Giard et Brière.

Worms, R. (1896): Organisme et société, Paris: Giard et Brière. 


\section{Die hauptsächlichen Periodika, die sich der Soziologie im eigentlichen Sinn widmen, sind:}

Revue internationale de sociologie

Annales de l'Institut international de sociologie

Année sociologique

Zeitschrift für Sozialwissenschaft

Rivista Italiana di Sociologia

American Journal of Sociology

\section{Zur Methode der Soziologie}

Comte, A. (1839-1842): Cours de philosophie positive, Paris: Bachelier; dt. Teilübersetzung: Die Soziologie. Positive Philosophie, Stuttgart: Kröner, 1974.

Durkheim, É. (2011 [1895]): Die Regeln der soziologischen Methode, hg. und übersetzt von René König, Berlin: Suhrkamp.

Mill, J. S. (2016 [1862]): System der deduktiven und induktiven Logik: Eine Darlegung der Prinzipien wissenschaftlicher Forschung, insbesondere der Naturforschung, Nachdruck, Norderstedt: Hansebooks.

\section{Zu speziellen Punkten:}

Bosco, A. (1898): La Statistica civile e penale e la reunione dell'instituto internazionale di statistica a Pietreburgo, Rom: Tipografia Nazignale di G. Bertero.

Langlois, Ch.-V. / Seignobos, Ch. (1898): Introduction aux études historiques, Paris: Hachette.

Steinmetz, S. R. (1894): Ethnologische Studien zur ersten Entwicklung der Strafe: nebst einer psychologischen Abhandlung über Grausamkeit und Rachsucht, Leiden: Doesburgh; Leipzig:

Harrassowitz.

Steinmetz, S. R. (1900): »Classification des types sociaux«, Année sociologique.

Tylor, E. B. (1888): »On a Method of Investigating the Development of Institutions applied to Laws of Marriage and Descent «, Journal of the Anthropological Institute of Great Britain and Ireland, Vol. XVIII, S. 245-270.

\section{INDEX}

Schlüsselwörter : Soziologie, Sozialwissenschaften, soziale Tatsache, Gesellschaft Mots-clés : sociologie, sciences sociales, fait social, société

\section{AUTEURS}

\section{PAUL FAUCONNET}

Paul Fauconnet war Soziologe. Nähere Informationen finden Sie hier. 


\section{MARCEL MAUSS}

Marcel Mauss war Soziologe, Ethnologe und Religionswissenschaftler. Nähere Informationen finden Sie hier. 